\title{
A theory of rock core-based methods for in-situ stress measurement
}

\author{
Kiyohiko Yamamoto* \\ Tsurugaya 1-26-15, Miyagino-ku, Sendai 983-0824, Japan \\ (Received November 2, 2004; Revised July 6, 2006; Accepted July 5, 2009; Online published November 30, 2009)
}

\begin{abstract}
The behavior of the inelastic strain of rocks under the loading of compression reflects the history of stresses applied to the rocks. A number of methods based on this rock property of stress memory have been proposed for measuring in-situ stress. The magnitudes of in-situ stress can be determined from drilled core samples by deformation rate analysis (DRA); in other words, rocks do have the property of in-situ stress memory. In general, the inelastic strain of rocks increases with an increase in applied stress difference. The Keiser effect observed in laboratory experiments is explained as the behavior of the inelastic strain of this well-known mode. However, this effect cannot be the mechanism of the in-situ stress memory because the effect does not potentially allow us to determine the magnitudes of previously applied stress. Here, I theoretically show that rocks exhibit another mode of inelastic strain under axial loading of compression-if locally concentrated stresses in rocks relax to some extent under in-situ stress at depth. The magnitudes of in-situ stress can be determined from the behavior of this mode of inelastic strain under axial loading. The results of DRA suggest that this hypothesis is actually valid and that it is not only valid for the DRA, but also for the other rock core-based methods used for measuring in-situ stress.
\end{abstract}

Key words: In-situ stress, stress memory, deformation rate analysis (DRA), stress measurement, inelastic strain, acoustic emission (AE), Kaiser effect.

\section{Introduction}

When uni-axial loading of compression is applied to a rock specimen in a laboratory, acoustic emission (AE) activity starts just at the point as which the applied stress exceeds the peak of stress previously applied to the specimen or the previous stress (e.g., Kurita and Fujii, 1979; Yoshikawa and Mogi, 1981). This phenomenon is known as the Kaiser effect. The memory of the previously applied stresses are found not only as the change in AE activity but also in the strain rate for rock specimens under uni-axial loading of compression at a constant loading rate (Kuwahara et al., 1990; Yamamoto et al., 1990). This property together with the Kaiser effect is called the rock property of stress memory.

Kuwahara et al. (1990) proposed a model for the inelastic deformation of rock specimens under axial loading of compression to explain the Kaiser effect. According to Kuwahara et al. (1990), there are many potential microcracks in a rock specimen, and the strengths of fracturing are specific to respective potential cracks in terms of applied stress to the specimen. If the applied stress is increased to a certain magnitude, all of the potential microcracks with fracture strengths smaller than the applied stress should move - that is, propagate or deform. When the applied stress is increased again after unloading, new microfractures hardly occur at first, and the preexisting cracks

* Retired from Graduate School of Science, Tohoku University, 2004.

Copyright (C) The Society of Geomagnetism and Earth, Planetary and Space Sciences (SGEPSS); The Seismological Society of Japan; The Volcanological Society of Japan; The Geodetic Society of Japan; The Japanese Society for Planetary Sciences; TERRAPUB. produced in the previous loading steadily move with an increase in applied stress until the applied stress reaches the peak of the previously applied stress. New micro-fractures begin to occur only after the applied stress has reached at the previous stress.

It is generally understood that the strength of potential cracks is determined not only by the axial stress but also by other stress components, such as confining pressure. Therefore, the behavior of micro-cracks under uni-axial loading should change depending not only on the previous axial stress but also on these other components of stress that have been acting during the time when the previous axial stress has been applied. Consequently, if the model by Kuwahara et al. (1990) is the only model that can explain the mechanism of the stress memory, the memory under uni-axial loading does not always have the same stress magnitude as the previous stress.

Since rock has been subjected to in-situ stresses for a long time on location, it may not be ridiculous to expect that rocks have some memories of the stress that they have been subjected to by the in-situ stresses. Kanagawa et al. (1977) and Yoshikawa and Mogi (1981) proposed the AE method and Yamamoto et al. $(1983,1990)$ proposed deformation rate analysis (DRA) as valid and reliable techniques for estimating in-situ stresses associated with the rock property of stress memory. The AE method and DRA were developed to detect the change in AE activity and the change in strain rate, respectively, for rock specimens under the uni-axial stress of compression being increased at a constant rate.

The stress memory found at the point at which the applied stress is equal to the absolute magnitude of in-situ stress is called the memory of in-situ stress from here on 
in this article. Directly associated with Kanagawa et al.'s (1977) suggestion that the magnitudes of the in-situ stress can be estimated by the AE method is the elementary question of whether or not rocks actually do have the property of in-situ stress memory. In order to provide an answer to this elementary question, Yamamoto et al. (1995) measured the memory of the vertical component of in-situ stress, that is, the memory of overburden pressure, by DRA to investigate this memory in relation to the sampling depth of the specimen. Based on their results, they concluded that rocks have the property of in-situ stress memory.

According to Yamamoto et al. (1990, 1995), if a cyclic uni-axial loading is repeated on a rock specimen at an interval of about 1 week, in-situ stress memory can be repeatedly found for that specimen. Yamamoto et al. (1997) pointed out further that in-situ stress memory can be found for rocks even after they have been left standing in air for a period of more than 1 year after their recovery. Seto et al. (1999) reported that the time interval, up to 7 years, does not strongly influence the in-situ stress determination by the AE method of repeated loading. On the other hand, Goodman (1963) demonstrated that the Kaiser effect in AE activity begins to fade out within a short time of about $10 \mathrm{~h}$ after the applied stress has been removed. Yamamoto (1991) reported that the memory of the previous stress applied experimentally, which is observed by DRA, shows the fading behavior similar to the Kaiser effect. These results suggest that insitu stress memory a long-term property, while the so-called Kaiser effect is a short-term one. In-situ stress memory has been defined as the memory that has the same magnitude as in-situ stress. The memory stress by the Kaiser effect does not necessarily have the same magnitude as the previous stress. Thus, we have as yet no convincing models for the property of in-situ stress memory.

In this paper, I briefly review DRA and present evidence proving that in-situ stress memory is one of the rock properties. I then propose a hypothesis to elucidate the property of in-situ stress memory. This hypothesis will show that DRA is one of the most logically acceptable methods for the estimation of in-situ stress magnitudes from rocks.

\section{In-situ Stress Memory}

\subsection{Deformation rate analysis}

Here, I use the term elastic strain to be that strain represented as a one-valued function of applied stress and the term inelastic strain to be that strain remaining after the removal of the elastic strain from the observed strain. The term inelastic strain rate means an increment in the inelastic strain for a unit increase in the applied stress when the applied stress is increased at a constant rate.

When a rock specimen is loaded by uni-axial stress of compression, many micro-fractures are induced in the specimen, generating AE events and producing micro-cracks. Kuwahara et al. (1990) modeled a micro-crack as a shear crack accompanied by tensile cracks, as proposed by Brace et al. (1966). This crack is produced by shear microfracturing. Deformation of the cracks is basically controlled by the shear displacement on a shear crack surface. The shear displacement of existing cracks increases and decreases against frictional resistance on the crack surface in
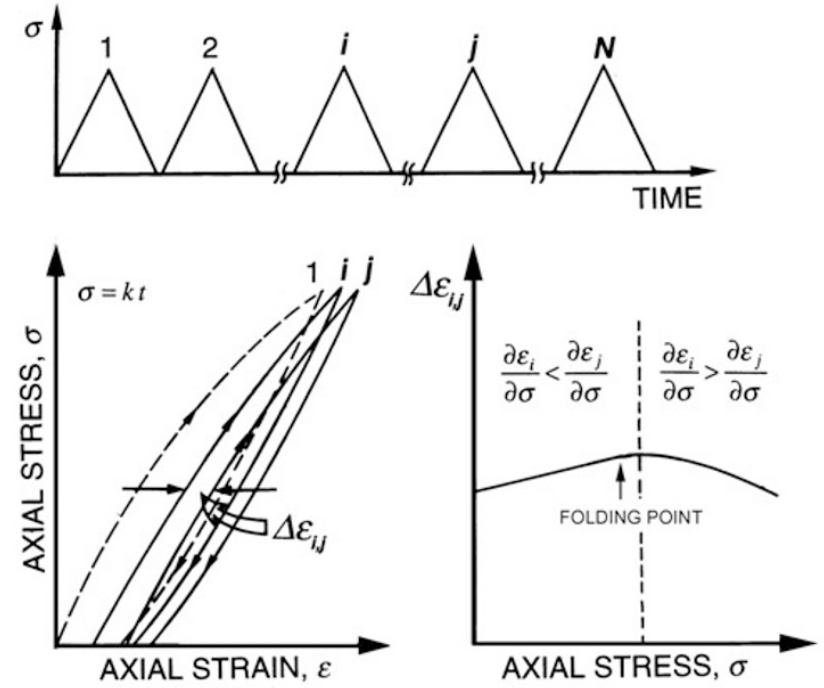

Fig. 1. Schematic illustration of the deformation rate analysis (DRA). The upper figure shows the axial stress $\sigma$ as a function of time applied for DRA. The lower left explains the definition of the strain difference function $\Delta \varepsilon_{i, j}(\sigma)$ on the stress-strain curves obtained by cyclic loading. The right schematically demonstrates the behavior of $\Delta \varepsilon_{i, j}(\sigma)$ and the definition of folding point.

order to release locally concentrated shear stress caused by the applied stress. This behavior of the cracks is thought to contribute to the behavior of inelastic strain during loading and unloading.

According to the above crack model, micro-fracturing produces an additional increment of inelastic strain. When no micro-fracture occurs, inelastic strain increases almost at a constant rate under an applied stress being increased at a constant rate because the displacement of existing shear micro-cracks is approximately proportional to the applied stress. Therefore, an increase in inelastic strain rate indicates the occurrence of micro-fracturing. If the Kaiser effect is observed in the inelastic strain behavior as well as in $\mathrm{AE}$ activity, the inelastic strain rate should begin to be higher at the point at which the applied stress is nearly equal in magnitude to the previous stress.

In order to detect the strain rate change, Yamamoto et al. $(1983,1990)$ proposed a function called the strain difference function $\Delta \varepsilon_{i, j}(\sigma)$, which is obtained by cyclically loading a specimen. The function $\Delta \varepsilon_{i, j}(\sigma)$ is defined for a pair of the $i$ th and the $j$ th loading cycle by

$$
\Delta \varepsilon_{i, j}(\sigma)=\varepsilon_{j}(\sigma)-\varepsilon_{i}(\sigma) \quad j>i .
$$

Here, $\varepsilon_{i}(\sigma)$ denotes the axial strain of a specimen at an applied stress $\sigma$ in the loading stage. Contraction and compression are defined to be positive in sign. $\varepsilon_{i}(\sigma)$ is continuously measured from the beginning of the first loading. The inelastic strain may include the components produced by a number of different causes. Some of these components may not vary with loading cycles, and these are removed from the strain difference function by Eq. (1) together with the linear and nonlinear components of elastic strain. Thus, the value of the function mainly represents the difference in the amount of inelastic strain at an applied stress between the $i$ th and the $j$ th loading cycles. 

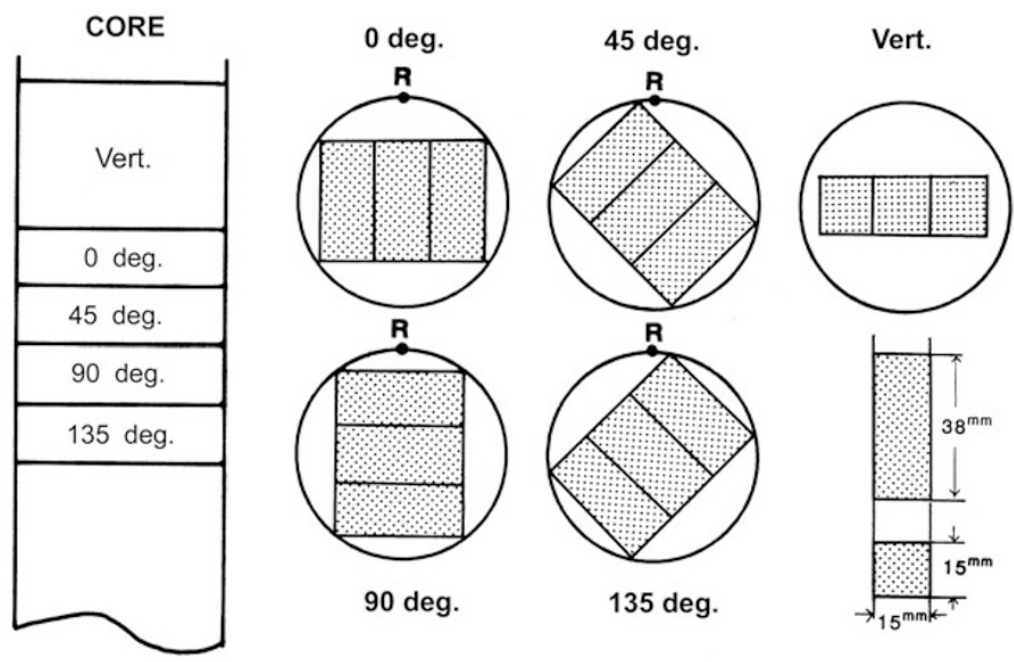

Fig. 2. Schematic illustration of the preparation of specimens from a core sample. In most cases, the specimens are sawed from a vertical core in order that the longer axes are taken in the vertical and in the four horizontal directions of azimuths at an interval of $45^{\circ} . R$ indicates the reference azimuth. The loading is performed along the longer axis.
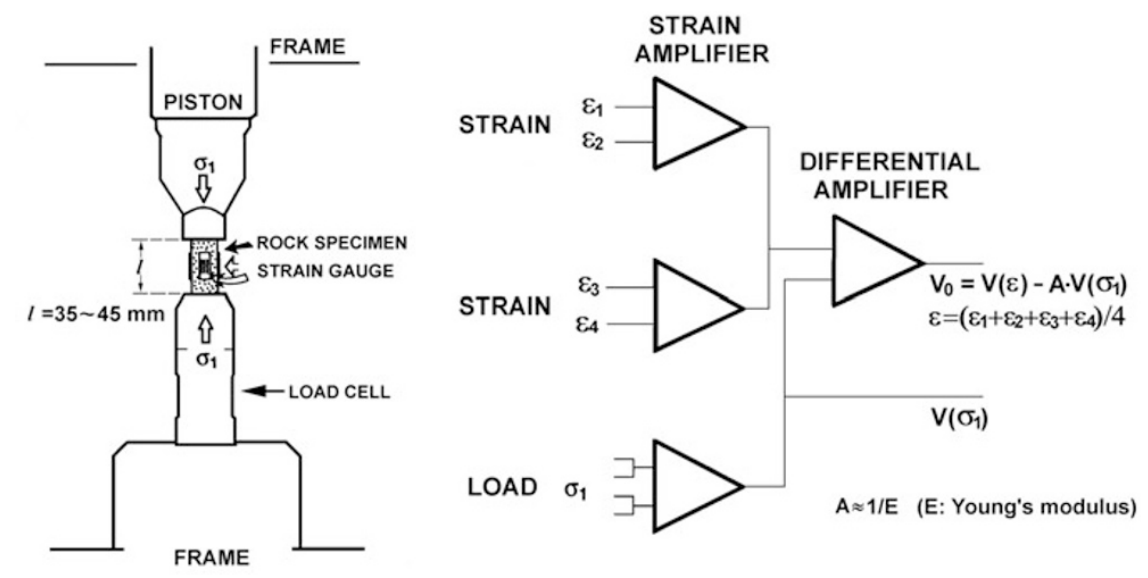

Fig. 3. Apparatus used for the deformation rate analysis. The left figure shows a specimen set in a loading machine. A strain gauge is pasted on each side of a rectangular specimen in order to measure the axial strain. The right figure illustrates instrumentation for measuring reduced strain. $\sigma_{1}$ denotes the axial applied stress and $\varepsilon_{i}$ the axial strain measured on each side of specimen.

The strain difference function is schematically illustrated in Fig. 1. The negative derivative of $\Delta \varepsilon_{i, j}(\sigma)$ with respect to $\sigma$ means that the inelastic strain rate in the $i$ th loading is larger than that in the $j$ th loading. According to the model proposed by Kuwahara et al. (1990), when applied stress is smaller than the previous stress, the function is approximately linear to the applied stress. However, when the applied stress is larger than the previous stress, the derivative of the function is negative. This latter effect is caused by two factors: (1) the micro-fractures begin to occur at the point at which the applied stress in the $j$ th loading reaches the previously applied peak stress and (2) the occurrence of the micro-fractures causes the inelastic strain rate to increase.

The previous stress magnitude may therefore be estimated as the magnitude of the applied axial stress at which the function begins to fold into the negative direction. The folding point is defined in principle as the point in the function at which the continuous decrease in its derivative starts, and folding stress or memory stress is the axial stress at the folding point. Using uni-axial loading of compression, Yamamoto et al. (1990) confirmed that the Kaiser effect is detectable by the strain measurement as well as by the measurement of AE activity.

In most cases of the measurement of in-situ stresses by DRA, axial strain is measured on specimens cut from a core sample in the vertical and in the four horizontal directions at an interval of $45^{\circ}$ in azimuth. Figure 2 illustrates such specimens. They are sawed from boring core samples in such a way that their axes lie along the vertical and the four azimuths. The specimens from their longer axes along the vertical are called vertical specimens, and those from their axes in the horizontal are called horizontal specimens. The memory stresses estimated from vertical and horizontal specimens are called the vertical and the horizontal stresses of memory, respectively. The data presented in this paper were obtained from the specimens of a rectangular prism with an approximate size of 15 (or 10) $\times 15$ (or 10) $\times 38$ (or 25) $\mathrm{mm}$ or from those of a cylinder with an approximate size of $18(\phi) \times 45 \mathrm{~mm}$. 
(a)

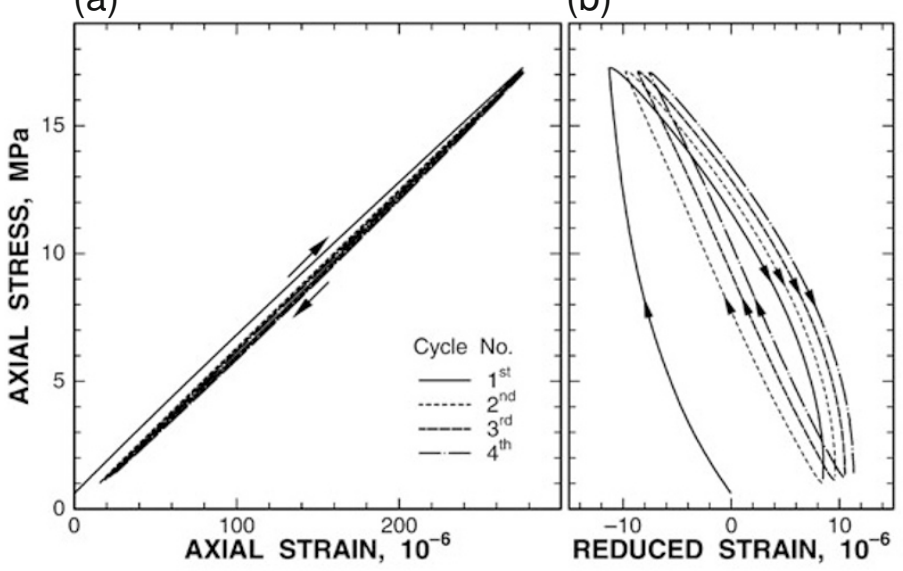

Fig. 4. Examples of an ordinary stress-strain curve and reduced stress-strain curve obtained from a siliceous slate specimen by four cycles of uni-axial loading. (a) Ordinary stress-strain curve. (b) Reduced stress-strain curve.

Loading is performed along the longer axis of specimen. The set-up for measuring strain is schematically illustrated in Fig. 3. Uni-axial stress of compression is cyclically applied at a constant increasing rate of between 3 and $5 \mathrm{MPa}$ per minute using a servo-controlled apparatus. The peak of applied stress is usually taken to be 1.5 - to 2.5 -fold higher than the target stress that has been estimated by the preliminary measurements.

In the case of hard rock specimens, of which the Young's modulus is $>50 \mathrm{GPa}$, the strain is required to be measured at a resolution $<10^{-7}$ in order to obtain the meaningful strain difference functions. The outputs of the four strain gauges are averaged to reduce those noises generated electrically and mechanically. The linear trend, of which the gradient is approximated to the Young's modulus of the specimen, is subtracted from the averaged strain in the way shown in Fig. 3 to detect the strain rate change with sufficient resolution. The strain remaining after the subtraction is called the reduced strain. Figure 4(a) and (b) shows the relations of the applied axial stress to the axial strain and to the reduced strain, respectively, measured on a specimen of granitic rock.

The outputs for the averaged strain and the reduced strain are digitized at $200 \mathrm{~Hz}$ sampling by a 12- or 16-bit A/D converter together with the output of the load. The number of sampling points reaches more than 80,000 at most in the loading stage per cycle. The number of data points is reduced to about 200 by averaging. This averaging procedure provides resolution of the strain difference functions smaller than the circuit noises of strain amplifiers. Therefore, the quality of data is almost the same either from the 12-bit or the 16-bit A/D converter. The strain difference functions are calculated using the reduced strain data thus processed.

\subsection{Modes of inelastic strain}

Using DRA, Yamamoto (1991) investigated the timeeffect of previously applied stresses on the deformation of specimens. Figure 5 shows an example of the results. The experiments were performed on vertical specimens from grano-diorite cores obtained from a depth of about $200 \mathrm{~m}$. Figure 5(a) schematically illustrates the loading history.
The first loading is performed to impress the previous stress of constant magnitude $\sigma_{\mathrm{p}}$ to specimens. $\tau_{0}$ is the duration time for applying the previous stress. After unloading the stress for the impression, the specimen is allowed to rest under ambient conditions for the period of $\tau$. The strain difference functions in Fig. 5(b) are obtained from the specimens that have been subjected to the same previous stress of $8 \mathrm{MPa}\left(\sigma_{\mathrm{p}}=8 \mathrm{MPa}\right)$, where $\tau_{0}$ is taken to be $1 \mathrm{~min}$ for all specimens and $\tau$ ranges from $10 \mathrm{~min}$ to $150 \mathrm{~h}$.

Let us denote the strain difference function $\Delta \varepsilon_{i, j}(\sigma)$ by $(i, j)$ for the sake of simplicity. The functions $(1,2)$ in Fig. 5(b) clearly fold near $8 \mathrm{MPa}$ in axial stress when $\tau$ is shorter than $1 \mathrm{~h}$. This is understood to be the Kaiser effect that is observed in inelastic strain behavior. The folding becomes obscure with the rest time $\tau$. The functions $(2,3)$ and $(3,4)$ do not fold near $8 \mathrm{MPa}$ but near $5 \mathrm{MPa}$ in axial stress, which is common for all cases of $\tau$. Although the gradient change near $5 \mathrm{MPa}$ in $(2,3)$ and $(3,4)$ is not as distinct as that observed near $8 \mathrm{MPa}$ in $(1,2)$, the degree of the folding appears invariable with $\tau$. This probably means that $(1,2)$ and the others respectively reflect the behavior of different modes of inelastic strain. Since the strain difference function represents the difference in inelastic strain behavior between two loading cycles in a pair, the difference implies that the predominant mode of inelastic strain is different between the first cycle and the other cycles of loading. It may therefore be concluded that the inelastic strain of rock specimens consists of at least two different modes.

If it is assumed that rocks have the property of in-situ stress memory, the folding in $(2,3)$ and $(3,4)$ may be considered to be caused by the in-situ stress memory, because the stress at the folding point is close to the magnitude of the overburden pressure. If this interpretation is correct, the results in Fig. 5 mean that in-situ stress memory remains after the first loading, while the Kaiser effect can be easily erased by the first loading. Further, the results suggest that in-situ stress memory is a long-term property, while the Kaiser effect is a short-term one. Since this article focuses on in-situ stress memory only, the folding point stresses observed in the strain difference functions from the pairs of 
a)

b)
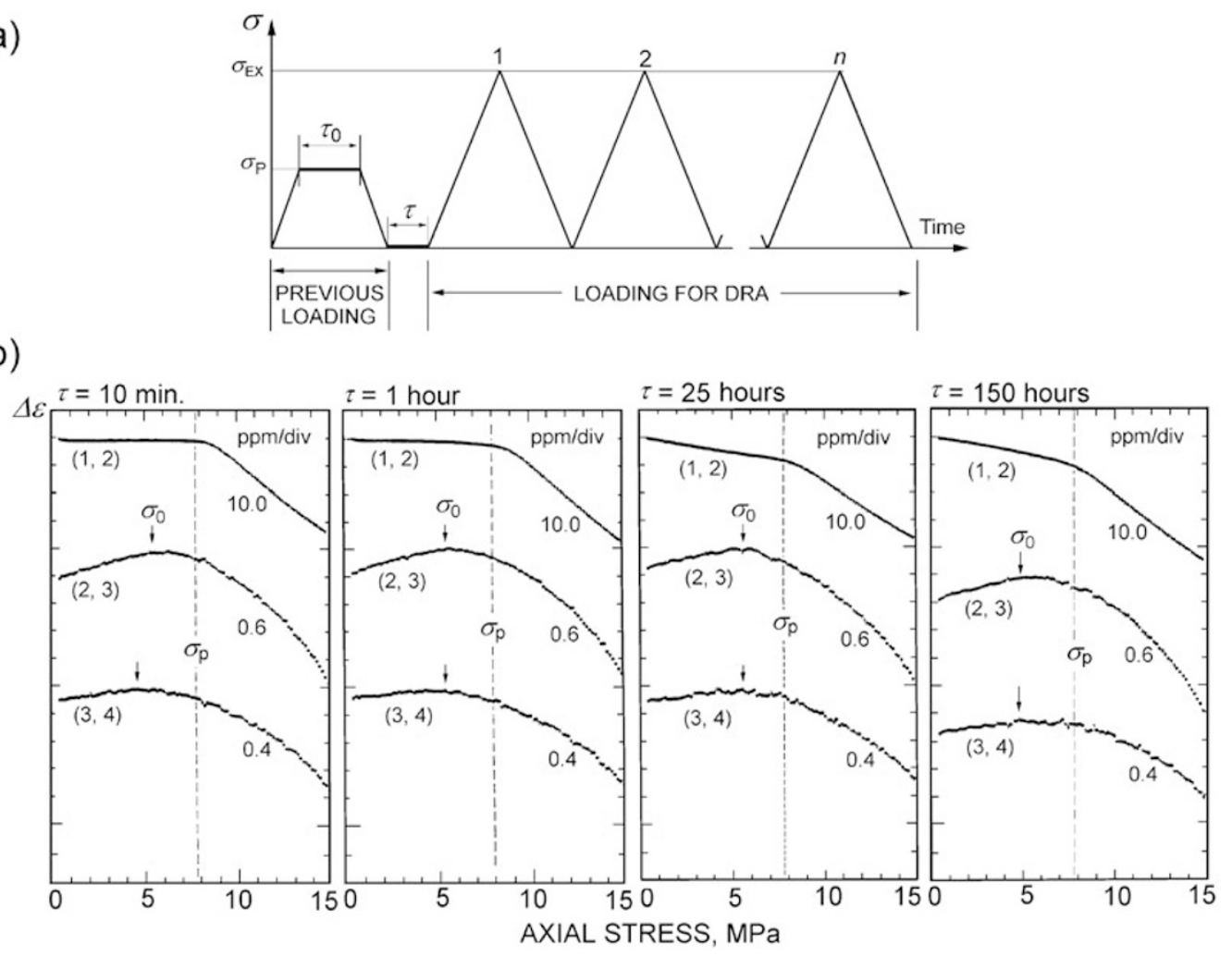

Fig. 5. Change in behavior of the strain difference function with loading history. (a) Axial stress as a function of time. $\tau_{0}$ and $\tau$ indicate the duration time of holding the axial stress constant and the duration time of specimens being kept under atmospheric pressure after unloading, respectively. $\sigma_{\mathrm{p}}$ and $\sigma_{\mathrm{ex}}$ are the previously applied stress and the peak of applied stress for DRA, respectively. (b) The change in the behavior with $\tau$ in the case of loading time $\tau_{0}=1 \mathrm{~min}$. Symbols $(i, j)$ are the same as $\Delta \varepsilon_{i, j}(\sigma)$. Sensitivity of the strain is denoted in $10^{-6} /$ division (ppm/div). Arrows are the folds that are taken as in-situ stress memory. The vertical offset given to the data of each $\Delta \varepsilon_{i, j}(\sigma)$ is arbitrary. (After Yamamoto, 1991).

loading cycles after the first are of concern. The rocks tested have probably been subjected to various kinds of temporal loads up to the time of measurement. The contamination to in-situ stress memory due to the temporal loads may have faded out during a time after the removal of the loads or have been wiped off by the first loading.

\subsection{Evidence for in-situ stress memory}

There may be a number of ways to verify the existence of the rock property of in-situ stress memory. One of these is to compare the memory stresses obtained by DRA with the in-situ stresses measured by established methods. Comparisons with an over-coring method have been carried out for depths shallower than about $20 \mathrm{~m}$. The results of the comparisons show that the stresses determined by DRA are in good agreement in terms of both orientation and magnitude with those obtained by the over-coring method (Koide et al., 1986). The overburden pressures at these depths are only about a few tenths of MPa. When the effect of the topography around the sites and the errors of stress measurement are taken into account, the depths can be considered to be too shallow to draw the conclusion that the measured magnitudes of memory stress are not the relative but the absolute ones of in-situ stress. For the reason, further investigations have been carried out for greater depths (Yamamoto et al., 1995).

Although over-coring methods and hydraulic fracturing techniques have been established as the "gold standards" for in-situ stress measurement, these methods are based on a number of assumptions. Their results may be more or less affected by the anisotropy of elasticity and the non-uniform distribution of the fracture-strength of rocks. The vertical in-situ stress is expected to have a magnitude equivalent to the overburden pressure, which can be estimated from the depth and the density of the overburden rocks. The effect of the topography near the site on the vertical stress may decrease with an increase in depth. The vertical stresses at great depths are thus expected to be the best standard reference for calibrating the measured stresses. If stress memory corresponds to in-situ stress, the vertical stress of memory should have a magnitude close to that of the overburden pressure at the sampling depth. Further, this should vertical stress of memory should be independent of the stress field regime at the site, reverse, strike-slip or normal fault regime.

Let us define a Cartesian coordinate system $(x, y, z)$ for a stress field and introduce a new coordinate system $\left(x^{\prime}, y^{\prime}, z^{\prime}\right)$ by rotating $(x, y, z)$ clockwise around $z$-axis by $\theta$. The normal stress $\sigma(\theta) \equiv \sigma_{x^{\prime} x^{\prime}}$ on the plane whose normal lies in the direction $\theta$ is expressed by,

$$
\begin{aligned}
\sigma(\theta) \equiv \sigma_{x^{\prime} x^{\prime}}= & \frac{1}{2}\left(\sigma_{x x}+\sigma_{y y}\right)+\frac{1}{2}\left(\sigma_{x x}-\sigma_{y y}\right) \cos 2 \theta \\
& +\sigma_{x y} \sin 2 \theta
\end{aligned}
$$

where $\sigma_{x x}, \sigma_{y y}$, and $\sigma_{x y}$ are the stress component in $(x, y, z)$. This expression holds for the arbitrarily oriented stress field. When the $z$-axis is taken vertically downward, 
Table 1. Densities and rock type of the cores obtained from the sites where the vertical memory stresses have been measured.

\begin{tabular}{llrccccc}
\hline Site & Depth $(\mathrm{m})$ & $N_{\mathrm{s}}$ & $\rho_{\mathrm{w}}\left(10^{3} \mathrm{~kg} / \mathrm{m}^{3}\right)$ & $\rho_{\mathrm{d}}\left(10^{3} \mathrm{~kg} / \mathrm{m}^{3}\right)$ & $\rho_{\mathrm{g}}\left(10^{3} \mathrm{~kg} / \mathrm{m}^{3}\right)$ & \multicolumn{1}{c}{$\phi(\%)$} & Rock type \\
\hline${ }^{1)}$ ABR & $30-100$ & 10 & $2.71 \pm 0.01$ & $2.70 \pm 0.01$ & $2.72 \pm 0.01$ & $0.9 \pm 0.1$ & granodiorite \\
${ }^{1)}$ YST & $77-78$ & 5 & $2.65 \pm 0.01$ & $2.64 \pm 0.01$ & $2.67 \pm 0.01$ & 0.8 & granodiorite \\
${ }^{2)}$ Ohtaki-2 & $331-1160$ & 6 & $2.64 \pm 0.03$ & $2.63 \pm 0.03$ & $2.66 \pm 0.01$ & $0.8 \pm 0.2$ & dasite, porphyrite, \\
& & & & & & \\
rhyolite, quartz diorite & & & \\
${ }^{2)}$ Ohtaki-3 & $376-1193$ & 3 & $2.66 \pm 0.02$ & $2.65 \pm 0.03$ & $2.68 \pm 0.01$ & siliceous sandstone, chert \\
${ }^{2)}$ Ohtaki-4 & $983-991$ & 2 & $2.77 \pm 0.01$ & $2.76 \pm 0.01$ & $2.77 \pm 0.01$ & 0.2 & siliceous slate \\
${ }^{2)}$ Ohtaki-5 & $469-475$ & 2 & $2.74 \pm 0.04$ & $2.72 \pm 0.04$ & $2.78 \pm 0.01$ & $2.2 \pm 0.2$ & siliceous slate \\
\hline
\end{tabular}

$N_{\mathrm{s}}$ : Number of specimens used for the density measurement; $\rho_{\mathrm{W}}$ : Wet bulk density; $\rho_{\mathrm{g}}$ : Dry bulk density; $\rho_{\mathrm{d}}$ : Density of matrix; $\phi$ : Porosity.

1) Data after Yamamoto et al. (1990). ${ }^{2)}$ Data after Yamamoto (1991).

Table 2. Rock type, apparent Young's modulus of specimens used for vertical stress measurement at respective depths.

\begin{tabular}{cllc}
\hline Depth $(\mathrm{m})$ & Site & Rock type & Apparent Young's modulus (GPa) \\
\hline 73 & ${ }^{1)}$ Esashi & granodiorite & $73-76$ \\
100 & ${ }^{1)}$ Esashi & granodiorite & $70-76$ \\
143 & ${ }^{1)}$ Yasato & granodiorite & $40-50$ \\
376 & ${ }^{2)}$ Ohtaki-3 & siliceous sandstone & $57-65$ \\
448 & ${ }^{2)}$ Ohtaki-2 & porphyrite & $65-78$ \\
475 & ${ }^{2)}$ Ohtaki-5 & siliceous slate & $55-58$ \\
600 & ${ }^{2}$ Ohtaki-3 & siliceous sandstone & $76-78$ \\
705 & ${ }^{2)}$ Ohtaki-4 & siliceous slate & $71-76$ \\
842 & ${ }^{2)}$ Ohtaki-2 & quartz diorite & $37-71$ \\
\hline
\end{tabular}

1) Data after Yamamoto et al. (1990). ${ }^{2)}$ Data after Yamamoto (1991).

the normal stress in the horizontal specimens should be expressed by a sinusoidal function of the azimuth $\theta$ with a pe$\operatorname{riod}$ of $\pi$. If the measured stress magnitudes are the memory of in-situ stresses, the stresses measured on the horizontal specimens can be fit on the curve of a sinusoidal function of azimuth. Although this is not the most direct way to prove that the magnitudes of the memory stress are the absolute ones of in-situ stresses, it does provide circumstantial evidence that rocks have the memory of a stress field or insitu stresses.

Another way to confirm that the magnitudes of in-situ stress memory is the absolute ones of in-situ stresses is to investigate the effect of pore pressure on the measured stresses because the in-situ stresses acting on a surface of rocks are sustained not only by the stresses of the solid part and but also by pore pressure. Therefore, the in-situ stress magnitudes should be equivalent to the sum of the integrated stresses over the solid part and the integrated pore pressure over the pore space. If the measured stress magnitudes are the absolute ones of in-situ stress memory in the solid part, the relationship among measured stress magnitudes, pore-pressure, and in-situ stress magnitudes is one of the keys that would verify that the measured stress magnitudes are the absolute magnitude. The pore pressure effect will be described in Section 4.3.

2.3.1 Identification of in-situ stress memory by DRA As described in Section 2.2, if in-situ stress memory exists, the reliable memory should be detected in the strain difference functions from the loading cycles that follow the first loading cycle. For this reason, uni-axial loading of three to five cycles has to be performed on each specimen to detect in-situ stress memory. The strain difference functions thus obtained do not always show such simple behavior as that schematically illustrated in Fig. 1, rather they often show curvilinear behavior even for the applied axial stress smaller than the folding stress. The curvilinear behavior makes the identification of memory difficult. Nevertheless, in most cases, the memory can be recognized as the fold that gives the largest change in the gradient of the function with respect to the applied axial stress. It may be noted that the folding point occasionally appears as if the gradient of the strain difference function is discontinuous at the folding point.

When more than one folding point of a similar degree is observed in a strain difference function, a number of conditions are imposed to choose one of them as the in-situ stress memory. If the specimens have been directed to the same direction at the same depth of the same site, the fold that corresponds to the in-situ stress should be observed at almost the same stress for all the specimens. Further, the folding stress should not be varied if the applied peak stress for DRA is varied. These are the auxiliary conditions for choosing the folding points as the in-situ stress memory of the direction. When the memories cannot be determined for a direction even following application of the above conditions, the determination may be performed on the additional condition that the memory stress magnitudes are expressed as a sinusoidal function of the azimuth. Although the determination of the folding stress using the last condition appears to be rather subjective, it is not easy to obtain the results from noises that consistently satisfy this condition.

2.3.2 Vertical stress Yamamoto et al. (1995) applied DRA to the vertical specimens from boring cores at six sites to measure the memory stresses at great depths of up to 

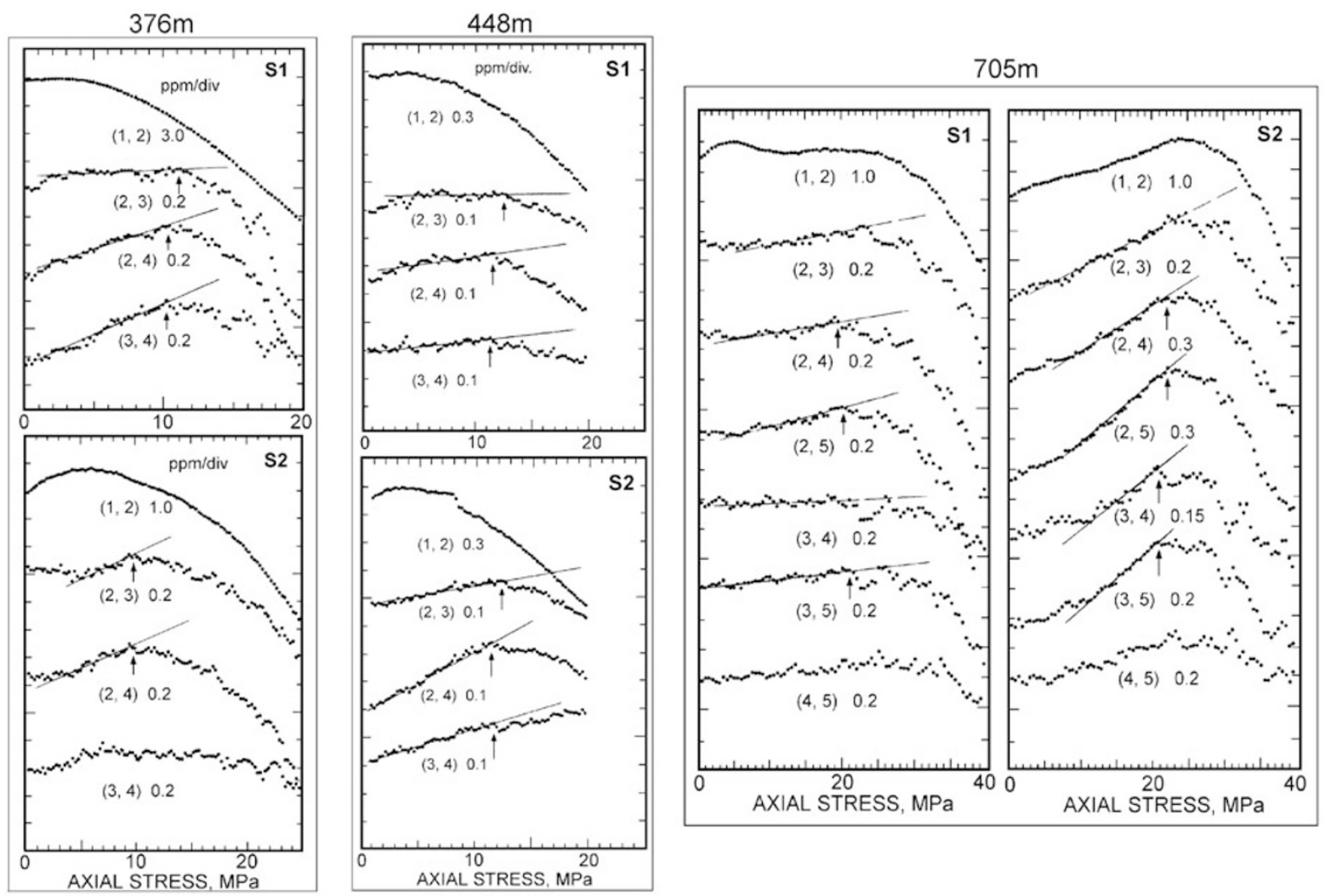

Fig. 6. Examples of strain difference functions $\Delta \varepsilon_{i, j}(\sigma)$ measured on vertical specimens obtained at depths of 376,448 , and $705 \mathrm{~m}$, respectively. Symbols S-1 and S-2 indicate specimen name. Other notations are the same as those in Fig. 5. This figure is reproduced from Yamamoto et al. (1995).

$842 \mathrm{~m}$. The cores were obtained at sites in three areas: Esashi (ABR) in northern Japan, and Yasato (YST) and Ohtaki (OT) in central Japan. The sites are known to be in the field of a strike-slip fault regime, with the exception of YST, which is located in a reverse fault regime (Yamamoto et al., 1990, 1997; Yamamoto, 1991). Average density and average porosity of the core samples are shown for the respective sites in Table 1. The specimens were soaked in water until the changing rate of the weight became about $10^{-5}$ per day. The saturated specimens were first weighed in air and in water, respectively, then dried in a vacuum vessel for the porosity measurement. The wet bulk density thus measured ranged from 2.64 to $2.77 \times 10^{3} \mathrm{~kg} / \mathrm{m}^{3}$, and the porosity was less than $1 \%$, with one exception of $2.2 \%$. The apparent Young's modulus and rock type of the specimens used for this measurement are shown in Table 2. The specimens are of hard rocks, as seen from the table.

Figure 6 illustrates an example of the strain difference functions obtained from the vertical specimens at three depths: 376,448 , and $705 \mathrm{~m}$. The peak of the applied stress for DRA can be seen to be about 20 and $25 \mathrm{MPa}$ for S1 and $\mathrm{S} 2$, respectively, at a depth of $376 \mathrm{~m}$ and $40 \mathrm{MPa}$ for both specimens at a depth of $705 \mathrm{~m}$. The stress memory corresponding to the overburden pressure can be found even in the functions of $(3,4)$ or $(3,5)$. The memories thus identified are marked with arrows in the Fig. 6. Linear lines are drawn as the reference to make the fold conspicuous. These lines are fitted by eye to the data in the relatively linear segments of strain difference curves, which appear at

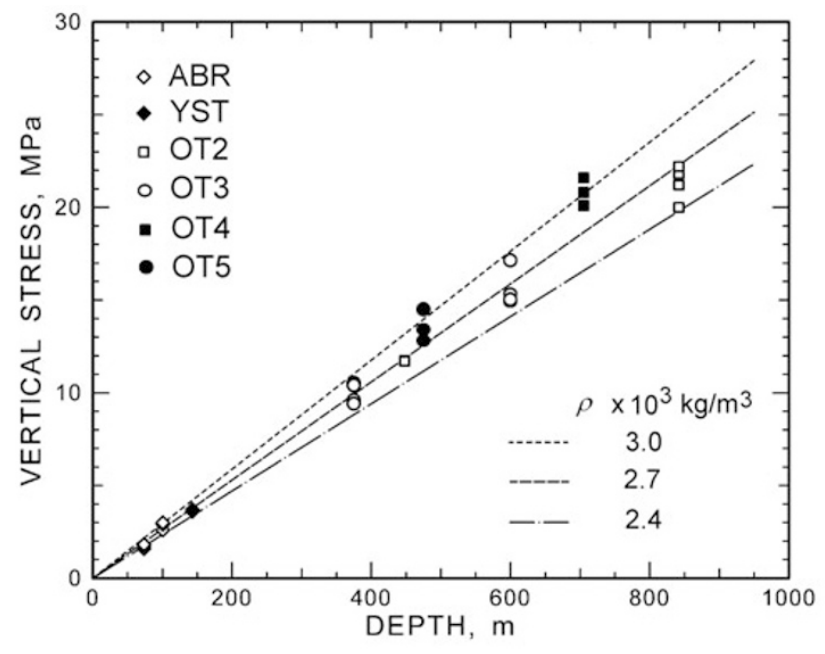

Fig. 7. Relation of vertical stress obtained by DRA to sampling depth. Straight lines denote the relation of overburden pressure to depth for the assumed average densities of overburden rocks. The same symbols indicate the data at the same sampling site. The data are the same as that in Yamamoto et al. (1995).

applied stresses smaller than the folding point. Even if the peak of applied stress to the respective specimens is different at $376 \mathrm{~m}$, the fold is found near 9.0 MPa in the axial stress. This stress magnitude is almost the same as the folding stresses for the other vertical specimens measured for a depth of $376 \mathrm{~m}$. The standard deviation for the memory 
stresses thus read is about $\pm 5 \%$ of their average at every depth.

The relationship between the vertical stress of memory and the sampling depth thus determined is shown in Fig. 7. Linear lines indicate the theoretical relations determined for a number of assumed values of average density for the overburden rocks, which are indicated in the figure. The average vertical stresses are seen to fall between the lines corresponding to densities of the overburden rocks of around $2.7 \times 10^{3} \mathrm{~kg} / \mathrm{m}^{3}$. These density values are close to those measured on core samples in Table 1 for both the stress fields of strike-slip and reverse fault regimes. Although the data may be affected by the topography near the sites, it can be seen that the vertical stresses of memory are almost consistent with their overburden pressures. This is the principal underlying reason for the conclusion drawn by Yamamoto et al. (1995) that rocks really do have the property of in-situ stress memory.

2.3.3 Horizontal stresses Figure 8 demonstrates the strain difference functions measured on the horizontal specimens of four azimuths at an interval of $45^{\circ}$. The functions for two sites, IKH351 and OT5.475, measured by
Yamamoto and Yabe (2001) and Yamamoto (1991) are shown in the upper and the lower frames of the figure, respectively. IKH351 indicates the functions for the granitic rocks recovered from a depth of $351 \mathrm{~m}$ at the Ikuha site, at a distance of about $3 \mathrm{~km}$ from the southwest end of the Nojima fault, Awaji Island, central Japan. The Young's moduli of the specimens are between 50 and $70 \mathrm{GPa}$. The average modulus for $\mathrm{R} 90^{\circ} \mathrm{E}$ is about $20 \%$ smaller than that for $\mathrm{R} 0^{\circ} \mathrm{E}$ (Yamamoto and Yabe, 2001). The specimens of OT5.475 are sawed from the boring cores recovered from a depth of $475 \mathrm{~m}$ at a site (OT5) in Ohtaki, Nagano Prefecture, Japan, close to the epicenter of the 1984 Nagano-ken Seibu earthquake $\left(M_{\mathrm{JMA}}=6.8\right)$. The rock type and physical constants are the same as those given in Tables 1 and 2.

For IKH351, four cycles of loading were performed on the two specimens of each azimuth for the DRA. The largest axial stress of each curve in every frame is approximately equal to the peak stress applied to the specimen when loading is performed for DRA. The peaks of the axial stress are slightly different between two specimens of each direction. The strain difference functions from the successive cycles without $(1,2)$ are shown in the figure. For (a)

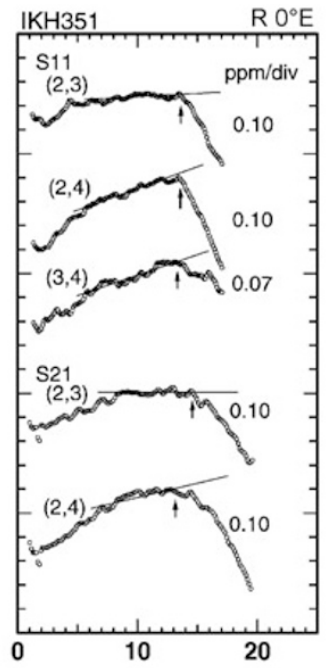

(b)

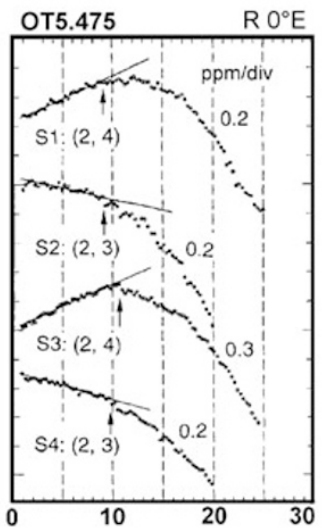

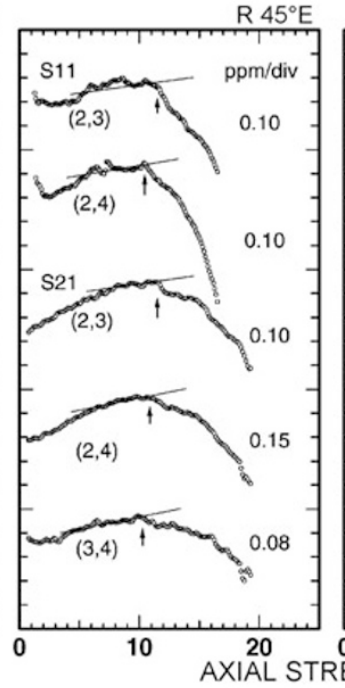
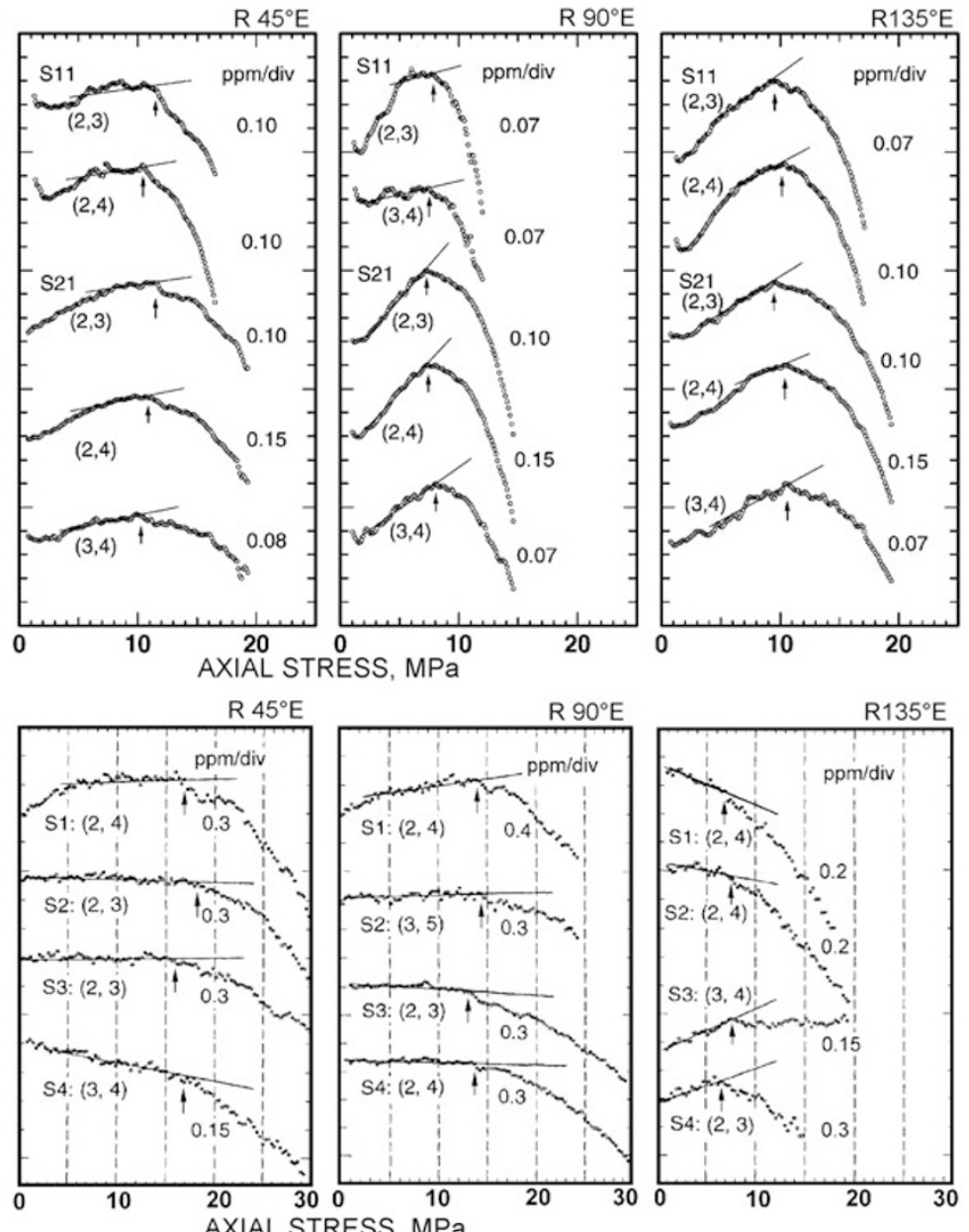

Fig. 8. Strain difference functions measured on the horizontal specimens of four azimuths at two sites. The records in each frame are of the same azimuth, indicated at the top of the frame. The references in the azimuth are arbitrary for the respective sites. $S_{i j}$ denotes the $j$-th measurement on the $i$-th specimen. Other notations are the same as those in Fig. 5. (a) For a depth of $351 \mathrm{~m}$ at the Ikuha site (IKH351) (after Yamamoto and Yabe, 2001). (b) For a depth of $475 \mathrm{~m}$ at the Ohtaki site (OT5.475) (after Yamamoto, 1991). 

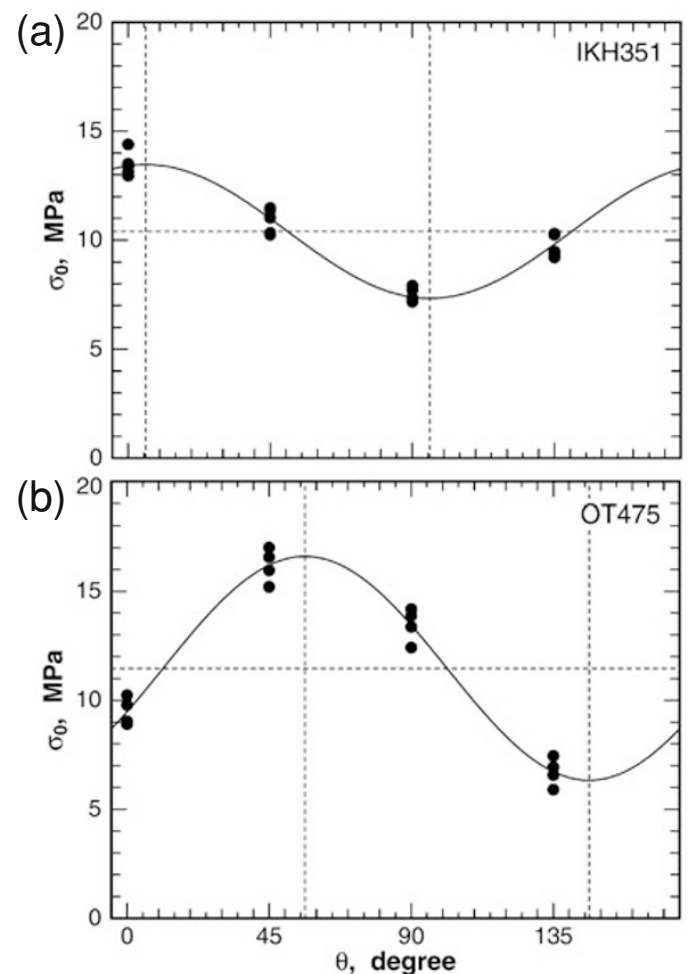

Fig. 9. The dependence of horizontal stresses on the azimuth. Solid circles denote the stress magnitudes read from the in-situ stress memories that are indicated by arrows in Fig. 8. The curves are the sinusoidal functions of azimuth fitted to the solid circles. (a) IKH351 (after Yamamoto and Yabe, 2001). (b) OT5.475 (after Yamamoto, 1991).

OT5.475, loading was cycled four or five times for each specimen. The strain difference functions in the figure are representative of each of the four specimens and azimuth. The arrows indicate the memories determined by eye. It can be seen in Fig. 8 that the folding point is repeatedly obtained from a specimen by cyclic loading and that the memory stresses of almost identical magnitude are obtained from the different specimens of the same depth and of the same direction. It can also be confirmed further from the frames for $\mathrm{R} 0^{\circ} \mathrm{E}, \mathrm{R} 90^{\circ} \mathrm{E}$, and $\mathrm{R} 135^{\circ} \mathrm{E}$ of $\mathrm{OT} 5.475$ that the folding stress is independent of the peak of the applied stress for DRA.

The memory stresses read near the arrows are plotted as a function of the azimuth in Fig. 9. The curves in this figure indicate the sinusoidal functions of azimuth fitted to the memory stresses. It can be seen that the memory stresses are well fitted to the curves, independently of the rock type of the specimens. This observation supports the interpretation that the memory stress represents a stress component in a stress field. Yamamoto et al. (1995) have already pointed out that the memory stress magnitudes are independent of the elastic property or of the anisotropy in the elasticity of specimens. Therefore, although the correlation between the elastic constants and the stresses is recognized for IKH351, this does not mean that the elastic anisotropy produces the apparent dependence of the memory stresses on the azimuth; rather, the correlation suggests the possibility of the elastic anisotropy being induced by the stresses in this case.
If memory stresses are identical to in-situ stresses, the memory stresses have to satisfy the conditions - that is, the vertical stress is nearly equal to the overburden pressure and the horizontal stress is expressed by a sinusoidal function with a period of $\pi$, as described in the previous section. Although the determination of stress memory may appear to be subjective, it is difficult to determine another set of such stress memories from the strain difference functions at each depth, as may be understood from the functions in Fig. 8. Some stress measurements have already been carried out by DRA. The measurements have confirmed that the memory stresses for horizontal specimens at a depth are well fitted by a sinusoidal function. For more examples of strain difference functions for horizontal specimens, I refer the read to earlier papers and reports (see Tamaki et al., 1991; Tamaki and Yamamoto, 1992; Yamamoto et al., 1995, 2004; Yamamoto and Yabe, 2001; Sato et al., 2003).

\section{Theory of In-situ Stress Measurement on Rocks 3.1 Models for two modes of inelastic strain}

Yamamoto (1995) proposed a hypothesis to verify the applicability of DRA to in-situ stress measurement. Here, I review the hypothesis and discuss the rock core-based methods for in-situ stress measurement from the viewpoint of this hypothesis in further detail. Based on the evidence described in Section 2.3, Yamamoto assumed two independent modes of inelastic strain in order to explain both the in-situ stress memory and the Kaiser effect. Let us denote applied axial stress and confining pressure for tri-axial loading by $\sigma_{1}$ and by $\sigma_{3}$, respectively, and the in-situ stress in the direction along the loading axis by $\sigma_{1}^{0}$. Inelastic strain is well known to increase with an increase in $\left(\sigma_{1}-\sigma_{3}\right)$. Here, this mode of inelastic strain is called the first mode (Mode-I). The inelastic strain of the other mode is associated with insitu stress memory and increases with an increase in the value of $\left|\sigma_{1}-\sigma_{1}^{0}\right|$-at least for $\sigma_{1}>\sigma_{1}^{0}$. This mode of inelastic strain is called the second mode (Mode-II).

Carlson and Wang (1986) and Meglis et al. (1991) investigated crack porosity under the ambient condition for rock samples recovered from depths. Crack porosity is known to increase with increasing sampling depth. These researchers attributed this property to the non-uniform expansion of constituent minerals due to the removal of in-situ stresses. This explanation suggests that the stress field in rocks under atmospheric pressure is non-uniform compared to that at depth, or that the stress field in rocks is more or less uniform at depths. Going a step further, this suggestion may enable the hypothesis to be framed as follows: the quasiuniform field of stress has been formed in rocks that are stable under in-situ stresses. Here, the quasi-uniform field means either the stress field excluding the stress concentrations that have the potential to generate micro-fractures in rocks or the stress field where the stress concentrations have partly relaxed.

Figure 10 schematically shows the enlarged parts of the models for the two modes of inelastic strain. Rocks are elastically heterogeneous. Respective blocks or constituent mineral grains of rock may deform differently under applied stresses. The blocks denoted $\mathrm{H}$ and $\mathrm{S}$ in the figure represent the hard and the soft homogeneous material, respectively, 
(a)

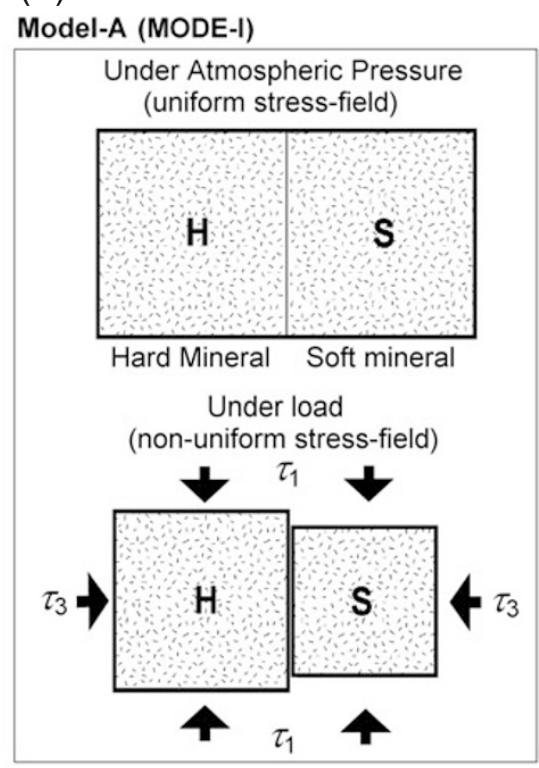

(b)

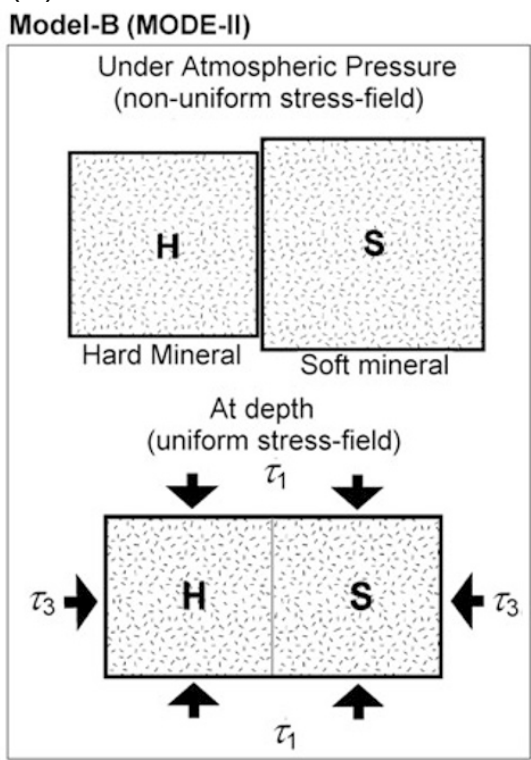

Fig. 10. Conceptual illustration of two composite models constructed to explain stress memories. H and S represent hard and soft constituents, respectively, of the composite. $\tau_{i}$ denotes a component of the stress applied to composites. (a) Model-A is for the inelastic strain of Mode-I. The constituents are welded each other in order not to cause misfits at their welded boundaries under atmospheric pressure. (b) Model-B is for the inelastic strain of Mode-II. The constituents are welded in order not to cause misfits at the welded boundaries under constant stresses $\tau_{i}$.

composing a rock. There may be two ways for the blocks that are separated each other to weld at a surface together into a composite material. One is to weld them under atmospheric pressure, and the other is to weld the blocks under stresses that have been separately deformed. The composites thus made are called Model-A and Model-B, respectively.

The stress field in Model-A may be uniform, and the surfaces of the blocks may adhere without misfits under atmospheric pressure. When the composite is subjected to applied stresses $\tau_{i}$, the blocks $\mathrm{H}$ and $\mathrm{S}$ may deform differently to generate misfits at the welded surface and to produce stress concentrations. The stress concentration may increase with increasing applied stress to cause a dislocation or fracture at the surface. Thus, Model-A may be taken as the model for the Kaiser effect or the inelastic strain of Mode-I. The stress field in Model-B is uniform under the initial stresses $\tau_{i}$, which are the stresses when the materials have been welded. When the initial stresses are removed from the composite, stress concentration may appear at the boundary surface. The stress concentration may increase with an increase in the initial stresses. In this model, those applied stresses that are different from the initial ones cause the stress concentration at the surface. This property may be expected for the quasi-uniform field under the initial stresses. Model-B may be thus taken as the model for the inelastic strain of Mode-II.

The stress fields and the inelastic deformation will be discussed hereafter for the quasi-uniform field under insitu stresses, when the specimens are loaded with uni-axial, tri-axial stresses or hydrostatic pressure. Let us define the stress deviation to quantify the stress concentrations. Here, the deviation is defined as the amount of the stress deviated from its average. An increase in the deviation may mean thus the increase in the stress concentrations having the potential to generate micro-fractures. For the rocks at the quasi-uniform state, stress concentrations may be produced, whenever the magnitudes of in-situ stress vary in a short time or the stresses different from the in-situ stresses are applied in a short time. It does not lose the generality of the following discussions to put the uniform stress field in Model-B in place of the quasi-uniform field.

\subsection{Stress in rock specimens under loading}

Rocks are aggregates of the mineral grains of various shapes that respectively have different elastic constants. The stress field in rocks is assumed to have been quasiuniform under in-situ stresses. In order to discuss the behavior of inelastic strain of Mode-II, I formulate here the stress field in specimens under compressive loading in terms of stress deviation. To do this, the followings are assumed: (a) the stress field for Mode-I and that for Mode-II are independent of each other; (b) the stress field in rocks is completely uniform under in-situ stresses; (c) inelastic strain increases with a growth in the stress concentration, and the stress concentration grows with an increase in the stress deviation; (d) the amount of inelastic strain produced is not so large as to affect the stress field in rock specimens.

Suppose a unit volume of rock surrounded by a surface $S$ of arbitrary shape. When the stresses at a position $\boldsymbol{x}$ in the rock is expressed by $\tau_{i j}(\boldsymbol{x})$, the average stress $\left\langle\tau_{i j}\right\rangle$ over the unit volume is defined by

$$
\left\langle\tau_{i j}\right\rangle=\int_{V} \tau_{i j}(\boldsymbol{x}) d v
$$

where $d v$ is a volume element. Using the divergence theorem (the Gauss theorem) and the equilibrium equation, 
Eq. (3) is generally expressed by

$$
\begin{aligned}
\left\langle\tau_{i j}\right\rangle= & \frac{1}{2} \int_{S}\left(x_{i} \check{T}_{j}^{*}(\boldsymbol{x})+x_{j} \check{T}_{i}^{*}(\boldsymbol{x})\right) d s \\
& +\frac{1}{2} \int_{V}\left\{x_{i} f_{j}(\boldsymbol{x})+x_{j} f_{i}(\boldsymbol{x})\right\} d v \\
\check{T}_{i}^{*}(\boldsymbol{x})= & \tau_{j i}^{*}(\boldsymbol{x}) v_{j}(\boldsymbol{x})
\end{aligned}
$$

Here, the summation convention is used. $\check{T}_{i}^{*}(\boldsymbol{x})$ and $f_{i}(\boldsymbol{x})$ are the surface traction on the boundary $S$ and the body force in the volume acting to the $x_{i}$-direction, respectively; $v_{j}(\boldsymbol{x})$ is the $x_{j}$-component of the surface normal of $d s$ at $\boldsymbol{x}$; $\tau_{j i}^{*}(\boldsymbol{x})$ is a stress component. This equation generally holds whenever the equilibrium equation holds everywhere in the volume. Equation (4) enables us to understand that the stress field in the volume is expressed as the superposition of the stresses produced by $\check{T}_{i}^{*}(\boldsymbol{x})$ applied on $S$ upon the stresses produced by $f_{i}(\boldsymbol{x})$ in the body.

For rocks in which there are no body forces from the outer fields, such as the gravitational force,

$$
\left\langle f_{i}(\boldsymbol{x})\right\rangle \equiv \int_{V} f_{i}(\boldsymbol{x}) d v=0
$$

$f_{i}(\boldsymbol{x})$ can be interpreted as the internal forces in this case. When $f_{i}(\boldsymbol{x})$ randomly distribute in space independently of their magnitudes, the second term of the right-hand side of expression (4a) vanishes. Since $x_{j} f_{i}(\boldsymbol{x})$ remain as they are after $\tau_{i j}^{*}(\boldsymbol{x})$ have been removed from the rock, the moments $x_{j} f_{i}(\boldsymbol{x})$ due to internal forces are interpreted to be the internal stresses equivalent to residual stresses caused by plastic deformation or dislocations.

Here, the stress field caused by randomly distributed internal stresses is expressed by $\tau_{i j}^{\mathrm{c}}(\boldsymbol{x})$. When macroscopically uniform stresses $\tau_{i j}^{*}$ are acting on $S$, there is a nonuniform stress field, $\tau_{i j}^{*}(\boldsymbol{x})$, in the volume caused by the non-homogeneity of the rocks. Since the stress field in a rock is the superposition of the field due to the stresses on $S$ and the field due to distributed internal stresses, $\tau_{i j}(\boldsymbol{x})$ may be written as

$$
\tau_{i j}(\boldsymbol{x})=\tau_{i j}^{*}(\boldsymbol{x})-\tau_{i j}^{\mathrm{c}}(\boldsymbol{x}) .
$$

Because the second term on the right-hand side of Eq. (4a) is always zero, $\left\langle\tau_{i j}^{\mathrm{c}}(\boldsymbol{x})\right\rangle$ should be always zero. The derivation of expression (4) and the justification of expression (5) are described in Appendix A.

For simplicity, $\tau_{i j}$ is written as follows: $\tau_{11}=\tau_{1}, \cdots$ $\cdot \cdot \tau_{23}=\tau_{4}, \cdot \cdot$. When the theory of the linear elasticity is applicable, $\tau_{i}^{*}(\boldsymbol{x})$ are proportional to $\left\langle\tau_{i}^{*}(\boldsymbol{x})\right\rangle=\tau_{i}^{*}$. Therefore, we may write as follows,

$$
\begin{aligned}
\tau_{i}^{*}(\boldsymbol{x}) & =b_{i j}(\boldsymbol{x}) \tau_{j}^{*} \\
\left\langle b_{i j}(\boldsymbol{x})\right\rangle & \equiv \int_{V} b_{i j}(\boldsymbol{x}) d v=\delta_{i j},
\end{aligned}
$$

where $\delta_{i j}$ is the Kronecker delta. Assumption (b) states that the stress field in a rock is completely uniform when $\left\langle\tau_{i}^{*}\right\rangle=\tau_{i}^{0}$. Putting $\tau_{i}^{*}=\tau_{i}^{0}$ and substituting expression (6) into expression (5), we obtain the following expression of $\tau_{i}^{\mathrm{c}}(\boldsymbol{x})$,

$$
\tau_{i}^{\mathrm{c}}(\boldsymbol{x})=b_{i j}(\boldsymbol{x}) \tau_{j}^{0}-\tau_{i}^{0}
$$

We can easily confirm that $\left\langle\tau_{i}^{\mathrm{c}}(\boldsymbol{x})\right\rangle=0$, and we can see that $\tau_{i}^{\mathrm{c}}(\boldsymbol{x})$ represent the residual stress field.

Specimens are prepared from the cored rock samples for measurements of in-situ stresses. The stress field of the specimens has been assumed to be completely uniform under the in-situ stresses. When macroscopically uniform stresses $\tau_{j}^{\mathrm{a}}\left(=\left\langle\tau_{j}^{\mathrm{a}}(\boldsymbol{x})\right\rangle\right)$ are applied to the specimen, by putting $\tau_{j}^{\mathrm{a}}$ in place of $\tau_{j}^{*}$ in expression (6) and substituting expression (7) into expression (5), we obtain the stress field $\tau_{i}^{\mathrm{II}}(\boldsymbol{x})$ for Mode-II as follows;

$$
\tau_{i}^{\mathrm{II}}(\boldsymbol{x})=b_{i j}(\boldsymbol{x})\left(\tau_{j}^{\mathrm{a}}-\tau_{j}^{0}\right)+\tau_{i}^{0}
$$

The stress field in the rock has been assumed above to be completely uniform under in-situ stresses. However, the experimental results in the previous section suggest that this is not completely realized. Otherwise, even if it is realized insitu, the stress concentration produced by the internal forces under atmospheric pressure has partly relaxed. The stress field $\tau_{i}^{\mathrm{I}}(\boldsymbol{x})$ for Mode-I is free from the internal forces. For these reasons, the stress field for Mode-I may be required to express the actual stress field in a specimen under applied stresses. The stress field is obtained by putting $\tau_{i}^{0}=0$ in expression (8), that is,

$$
\tau_{i}^{\mathrm{I}}(\boldsymbol{x})=b_{i j}(\boldsymbol{x}) \tau_{j}^{\mathrm{a}}
$$

In this study, it has been assumed that the stresses $\tau_{i}^{\mathrm{T}}(\boldsymbol{x})$ in a specimen under applied stresses $\tau_{i}^{\mathrm{a}}$ are expressed by the sum of the stresses for Mode-I and for Mode-II, that is,

$$
\tau_{i}^{\mathrm{T}}(\boldsymbol{x})=(1-\eta) \tau_{i}^{\mathrm{I}}(\boldsymbol{x})+\eta \tau_{i}^{\mathrm{II}}(\boldsymbol{x}) ; \quad \text { for } \eta \leq 1,
$$

where $\eta$ denotes a constant to be experimentally determined.

The main aims of this paper are to show that rocks have the memory of in-situ stresses and to briefly review the potential of the existing methods to precisely measure in-situ stress magnitudes from the present hypothesis. To this end, it is most essential to investigate the behavior of inelastic strain of Mode-II in relation to applied stresses $\tau_{i}^{\mathrm{a}}$, even if expression (10) has not been established.

\subsection{Stress deviation in rock specimens under loading}

Let us define the stress deviation $\delta \tau_{i}(\boldsymbol{x})$ for Mode-II by

$$
\delta \tau_{i}(\boldsymbol{x})=\tau_{i}^{\mathrm{II}}(\boldsymbol{x})-\tau_{i}^{\mathrm{a}}
$$

Making use of expression (8) and the second equation of (6), the deviation is written by

$$
\begin{aligned}
\delta \tau_{i}(\boldsymbol{x}) & =a_{i j}(\boldsymbol{x})\left(\tau_{j}^{\mathrm{a}}-\tau_{j}^{0}\right), \\
\left\langle a_{i j}(\boldsymbol{x})\right\rangle & =0 \\
a_{i j}(\boldsymbol{x}) & \equiv b_{i j}(\boldsymbol{x})-\delta_{i j}
\end{aligned}
$$

where the average \langle\rangle is taken over a unit volume of a specimen.

Based on assumption (c), inelastic strain may increase with increasing absolute values of $\delta \tau_{i}(\boldsymbol{x})$. In order to discuss the inelastic strain behavior of rock specimens under loading of compression, I define the degree of deviation $I_{i}$ 
by

$$
\begin{aligned}
I_{i} & =\int_{V}\left\{\delta \tau_{i}(\boldsymbol{x})\right\}^{2} d v=A_{i j k}\left(\tau_{j}^{\mathrm{a}}-\tau_{j}^{0}\right)\left(\tau_{k}^{\mathrm{a}}-\tau_{k}^{0}\right) \\
A_{i j k} & =\int_{V} a_{i j}(\boldsymbol{x}) a_{i k}(\boldsymbol{x}) d v
\end{aligned}
$$

where $V$ is a unit volume of a specimen.

Although the influence coefficients $a_{i j}(\boldsymbol{x})$ could be exactly determined only when the spatial distribution and intensity distribution of the internal forces was specified, it is seen by making use of expression (12b) that at least the following relationship generally holds,

$$
A_{i j j}>0 \text { and } A_{i j j}>A_{i j k}, \text { for } j \neq k \text {. }
$$

If it is assumed that the coefficient $a_{i j}(\boldsymbol{x})$ is completely independent of $a_{i k}(\boldsymbol{x})$ for $j \neq k$, the following inequality holds,

$$
A_{i j j} \gg A_{i j k}, \quad \text { for } j \neq k .
$$

Using condition (15), $I_{i}$ is approximated by

$$
I_{i} \approx A_{i j j}\left(\tau_{j}^{\mathrm{a}}-\tau_{j}^{0}\right)^{2} .
$$

Equation (16) is fundamental for the stress deviation related to the inelastic strain of Mode II.

\section{Methods for Detection of In-situ Stresses}

The rock-core based methods for measurement or detection of in-situ stresses are classified into two groups: (1) those using axial loading; (2) those using the loading of hydrostatic pressure. The latter includes the method using the time-dependent property of deformation under atmospheric pressure. The deformation rate analysis (DRA) and the acoustic emission (AE) method are classified into the former group and the differential strain curve analysis (DSCA) and anelastic strain recovery method (ASR; e.g., Enever and Mckay, 1976) are considered part of the latter group. In the following sections, these methods for in-situ stress measurement are discussed within the context of the present hypothesis.

\subsection{Method using axial loading}

When an axial stress is applied to a rock specimen, stresses for Mode-I are superposed on those for Mode-II, as described above. The stress deviation for Mode-I may monotonically increase with an increase in applied stress, or applied stress difference in the case of axial loading, because it is caused by elastic heterogeneity and the irregularity of the grain shape of constituent minerals. The inelastic strains of Mode-I are therefore expected to monotonically increase with an increase in the applied stress difference.

The stress deviation for Mode-II under uni-axial or triaxial compression is obtained by putting

$$
\tau_{1}^{\mathrm{a}}=\sigma_{1}>\tau_{2}^{\mathrm{a}}=\tau_{3}^{\mathrm{a}}=\sigma_{3}=p_{\mathrm{c}}, \quad \tau_{4}^{\mathrm{a}}=\tau_{5}^{\mathrm{a}}=\tau_{6}^{\mathrm{a}}=0,
$$

where $\sigma_{i}$ denotes the axial stress applied in compressive loading, and $p_{\mathrm{c}}$ denotes the confining pressure. Therefore, $p_{\mathrm{c}}=0$ indicates the case of uni-axial loading. The degree of stress deviation is written from expression (16) as

$$
\begin{aligned}
& I_{i} \approx A_{i 11}\left(\sigma_{1}-\tau_{1}^{0}\right)^{2}+c_{i} \\
& c_{i}=A_{i j j}\left(\tau_{j}^{\mathrm{a}}-\tau_{j}^{0}\right)^{2}, \quad \text { for } j \neq 1
\end{aligned}
$$

Here, $c_{i}$ is independent of $\sigma_{1}$ and is thus constant for $p_{\mathrm{c}}$ fixed. It is seen from this expression that $I_{i}$ takes its minimum at $\sigma_{1}=\tau_{1}^{0}$ for every number of $i$. This means that the stress distribution in a specimen is the most uniform at $\sigma_{1}=\tau_{1}^{0}$, not only for the component of $\tau_{1}\left(=\tau_{11}\right)$ but also for all of the stress components.

For the stress deviation for Mode-II, the inelastic strain is expected to behave as follows: an amount of inelastic strain becomes larger with an increase in $I_{i}$. Since the inelastic strain is considered to have the same sign as the elastic strain caused by the stress $\left(\sigma_{1}-\tau_{1}^{0}\right)$, a specimen may expand longer than that elastically expected when $\sigma_{1}$ is decreased from $\tau_{1}^{0}$; alternatively, it may become shorter than that elastically expected when $\sigma_{1}$ is increased from $\tau_{1}^{0}$.

Let us take a shear crack accompanied by tensile cracks as a model of micro-cracks in rock specimens under axial loading of compression (e.g., Brace et al., 1966; Kuwahara et al., 1990) and suppose that the relative displacement on the shear crack surfaces is zero at $\sigma_{1}=\tau_{1}^{0}$. The displacement increases with a decrease in $\sigma_{1}$ from $\tau_{1}^{0}$. When $\sigma_{1}$ is increased from zero, the displacement decreases to zero at $\sigma_{1}=\tau_{1}^{0}$, at which point these cracks are locked and new cracks are produced for $\sigma_{1}>\tau_{1}^{0}$ because accompanying tensile cracks are completely closed at $\sigma_{1}=\tau_{1}^{0}$. This suggests that the cracks that contribute to the inelastic strain for $0<\sigma_{1}<\tau_{1}^{0}$ are different from the cracks that contribute to the inelastic strain for $\tau_{1}^{0}<\sigma_{1}$.

A problem in in-situ stress estimation is how to detect $\tau_{1}^{0}$ in the inelastic strains composed of Mode-I and Mode-II. The DRA will be discussed below.

4.1.1 Deformation rate analysis The behaviors of inelastic strains of Mode-I and Mode-II and strain difference functions are inferred from the above discussions. The inferred behaviors of the functions are shown in Fig. 11(a) and (b), where it is assumed that the apparent stiffness for Mode-II does not change for $0<\sigma_{1}<\tau_{1}^{0}$ but does increase for $\tau_{1}^{0}<\sigma_{1}$ with every loading cycle. In this context, the apparent stiffness means an increment of the applied stress necessary to produce a unit increment of strain. Figure 11(a) shows the inelastic strains of Mode-I and ModeII, and Fig. 11(b) shows the strain difference functions for $\eta=0.5$. The figures suggest that the observed behaviors of inelastic strain can be theoretically simulated by the behaviors of the two modes of inelastic strains. See Appendix B for the discussions in some details.

For the first approximation, it is adequate to consider that the inelastic strain of Mode-I and that of Mode-II are independent each other. The inelastic strain of Mode-I is responsible for the strength of specimens, while that of Mode-II is not-at least not directly-responsible for it. When $\tau_{1}^{0}$ becomes close to the strength of a specimen for axial stress of compression, the curvilinear behavior of the inelastic strain of Mode-I may become conspicuous, even in the later cycles of loading. Therefore, it is easily inferred 
a)

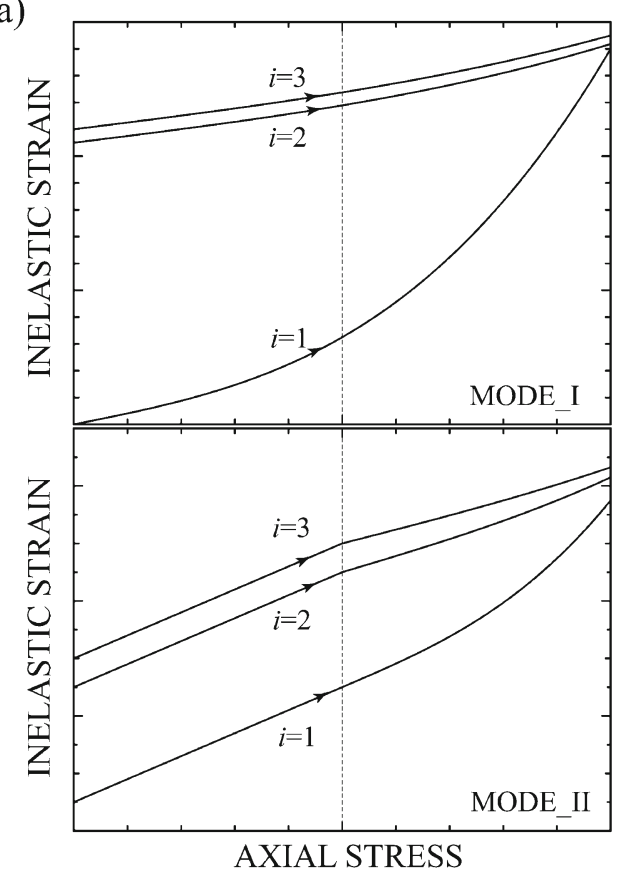

b)

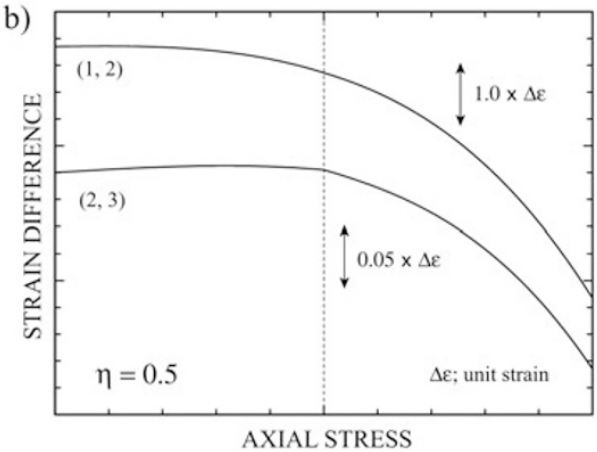

Fig. 11. (a) The inferred inelastic strain behaviors for composites under successive uni-axial loading. The upper and the lower figures show the behaviors theoretically inferred for Mode-I and Mode-II, respectively, when the applied axial stress $\sigma_{1}$ is increased. $i$ is the cycle number of loading. $\sigma_{1}^{0}$ is the component of applied stress that makes the deviation of stress field in the composite minimum. (b) The inferred behaviors of strain difference function for $\eta=0.5$. The vertical scales and the vertical offsets are arbitrary.

that the identification of the in-situ stress memory by DRA becomes difficult as in-situ stress approaches the strength of a specimen for axial loading.

It is well known that the strength of a rock specimen for axial loading of compression increases with an increase in confining pressure. The stress deviation expressed by Eq. (18) implies that in-situ stress magnitudes of memory are independent of confining pressure-when the confining pressure is kept constant. According to this hypothesis, the memory may be found in the inelastic deformation behavior of a specimen under tri-axial loading of compression, even when the in-situ stress is larger than the strength of a specimen for the uni-axial loading of compression. One of the future problems facing researchers is to develop the technology for measuring stress memory under tri-axial loading.

\subsection{Methods using hydrostatic pressure loading}

$I_{i}$ for a specimen under the hydrostatic pressure $p$ is obtained by putting

$$
\tau_{1}^{\mathrm{a}}=\tau_{2}^{\mathrm{a}}=\tau_{3}^{\mathrm{a}}=p \text { and } \tau_{4}^{\mathrm{a}}=\tau_{5}^{\mathrm{a}}=\tau_{6}^{\mathrm{a}}=0
$$

in expression (14). $I_{i}$ for the specimen is expressed by

$$
\begin{aligned}
I_{i} & \approx \sum_{j=1}^{3} A_{i j j}\left(p-\tau_{j}^{0}\right)^{2}+c_{i} \\
c_{i} & =\sum_{j=4}^{6} A_{i j j}\left(\tau_{j}^{0}\right)^{2}
\end{aligned}
$$

Here, $c_{i}$ is constant with respect to $\sigma_{1}$, and further $c_{i}$ is constant independent of the stress component, at least when the theory of linear elasticity is valid. Expression (20) is invariable with regard to the rotation of the coordinate system. $I_{i}(i=1,2,3)$ can be thus be taken as the degree of deviation for the normal stress component of an arbitrary direction. Here, $p=0$ means the stress-free state.

Differential strain curve analysis (e.g., Montgomery and Ren, 1981), which is based on DSA (Simmons et al., 1974), has been developed as a method for estimating insitu stresses. In DSCA, the longitudinal strains in more than six directions are measured on a specimen as the functions of applied hydrostatic pressure $p$, and in-situ stresses are estimated from the magnitudes of non-linear strain components at $p=0$. It is inferred from the paper by Meglis et al. (1991) that DSCA is based on the rock property of the non-uniform expansion of constituent minerals caused by the removal of in-situ stresses. This basic concept may be identical to that for DRA. Therefore, the validity of DSCA as a method for estimating in-situ stress may be discussed in terms of $I_{i}$ as well.

The stress deviation $I_{i}$ in a specimen at $p=0$ is generally expressed by the combination of the terms of $\left(\tau_{j}^{0}\right)^{2}$. In order that in-situ stresses are successfully estimated by DSCA, at least two conditions may be required in the present context: (1) that the relations of $I_{i}$ to $\tau_{i}^{0}$ or the relative values of $A_{i j j}$ in Eq. (20) are explicitly represented, because $I_{i}$ is expressed as the combination of all in-situ stress components; (2) that the constitutive relation between $I_{i}$ and the amount of inelastic strain is known.

Here, we investigate the relation of $I_{i}$ to $\tau_{i}^{0}$ for further discussion. By putting

$$
\begin{aligned}
G_{i} & =A_{i 11}+A_{i 22}+A_{i 33} \\
H_{i} & =A_{i 11} \tau_{1}^{0}+A_{i 22} \tau_{2}^{0}+A_{i 33} \tau_{3}^{0},
\end{aligned}
$$


it is seen that $I_{i}$ reaches its minimum at

$$
p=p_{i}=H_{i} / G_{i} .
$$

The integrand for $A_{i j j}$ is positive either for $i=j$ or for $i \neq j(j=1,2,3)$. Therefore, the two extreme cases will be investigated as follows: One is the case of

$$
A_{i i i} \gg A_{i j j}, \quad \text { for } i \neq j,
$$

and the other is the case of

$$
A_{i i i}=A_{i j j}, \quad \text { for } i, j=1,2 \text {, and } 3 \text {. }
$$

In the case of expression (23), $I_{i} \approx A_{i i i}\left(\tau_{i}^{0}\right)^{2}+c_{i}$ at $p=0$, and $I_{i}$ decreases to its minimum $c_{i}$ at $p=\tau_{i}^{0}(i=$ 1,2 , and 3) as $p$ is increased from zero. It is obvious that, in principle, $\tau_{i}^{0}$ can be determined, if the constitutive relation between $I_{i}$ and the inelastic strain has been established. This is considered to be one of the possible explanations of DSA or DSCA, when they are successful. A similar explanation may be possible for the method of ASR because the internal stresses in the second term of the right-hand side of expression (4a) are considered to be the sources of anelastic strain or time-dependent inelastic strain as well.

Attention should be paid to the fact that $I_{i}$ decreases to its minimum at $p=\tau_{i}^{0}$, when the applied hydrostatic pressure to a specimen is increased. If we assume that the longitudinal component $\varepsilon_{i}^{\text {inel }}$ of inelastic strain is proportional to $I_{i}^{1 / 2}(i=1,2,3), \varepsilon_{i}^{\text {inel }}$ changes its behavior from a decrease to an increase at $p=\tau_{i}^{0}$. This suggests the possibility of finding in-situ stress memories without having exact knowledge of the constitutive relation. However, it seems that condition (23) is much more difficult to satisfy than the condition (15) that is required for DRA.

In the latter case of $A_{i i i}=A_{i j j}, I_{i}(i=1,2,3)$ has the same value independent of $i$, when $p=0$. This implies that the magnitude of each component of in-situ stress cannot be determined, even if the non-linear components of strains at $p=0$ have been precisely measured and the constitutive relation has been established. $I_{i}$ has its minimum at

$$
p=p_{i}=\left(\tau_{1}^{0}+\tau_{2}^{0}+\tau_{3}^{0}\right) / 3
$$

for any component of stress. This means that the apparent compliance of specimens achieves its minimum at the same pressure for all the components of stress. Therefore, the magnitudes of the respective components of in-situ stress cannot be estimated by monitoring the apparent compliance change. Here, the apparent compliance means an increment in strain for a unit increment of applied stress. It holds - without assumptions and unconditionally - that the average of $I_{i}$ over $i$ increases with an increase in $\tau_{j}^{0}$ under atmospheric pressure. Since the average of $\tau_{j}^{0}$ generally increases with an increase in depth, the average of $I_{i}$ under the atmospheric pressure increases with an increase in the sampling depth. The behavior of crack porosity observed by Carlson and Wang (1986) and Meglis et al . (1991) is consistent with this increase in $I_{i}$, with the only assumption being that the crack porosity increases with an increase in $I_{i}$. In an actual measurement, if the magnitude of $p$ that makes $I_{i}$ minimum is found for a direction, the magnitude of $p$ may lie between the in-situ stress magnitude in the direction and the average in-situ stress given by expression (25).

In order to determine a stress field, it is necessary to measure more than six components of stress. To do this by the methods using axial loading, the measurements have to be performed at least six times; in contrast, only one measurement is needed by the methods using hydrostatic loading. This is the advantage of the methods using hydrostatic loading. However, it has not yet been decided which modelthat expressed by (23) or that by (24) - approximates the actual state of rocks. This may a problem to be studied later.

\subsection{Pore pressure effect}

From the definition of the average stresses, the average stresses $\left\langle\tau_{i j}\right\rangle$ over a unit volume of a rock specimen, including fluid pores of porosity $\phi$ may be expressed by

$$
\left\langle\tau_{i j}\right\rangle=(1-\phi)\left\langle\tau_{i j}^{\mathrm{s}}\right\rangle+\phi \delta_{i j} P_{\mathrm{p}} .
$$

Here, $\delta_{i j}$ means the Kronecker delta, and the positive sign of stress denotes compression. This expression holds without any assumption-i.e., it is independent of pore shape and orientation.

When rocks are subjected to in-situ stress $\left\langle\tau_{i j}^{0}\right\rangle$, the stress in the rocks may be expressed by

$$
\left\langle\tau_{i j}^{0}\right\rangle=(1-\phi)\left\langle\tau_{i j}^{\mathrm{s} 0}\right\rangle+\phi \delta_{i j} P_{\mathrm{p}}
$$

Here, $\left\langle\tau_{i j}^{\mathrm{s} 0}\right\rangle$ is the average stress in the matrix or solid part of rocks. Since we assumed the stress in rocks to be quasiuniform under in-situ stresses, the stress in the solid part of rocks can be considered to be quasi-uniform at the stresses $\left\langle\tau_{i j}^{\mathrm{s} 0}\right\rangle$. In loading tests in laboratories, pore pressure can be taken to be equal to atmospheric pressure if rock specimens have been dehydrated or the pores can be regarded to be interconnected to each other. In this case, as seen from expression (27), stress deviation may be the minimum at

$$
\left\langle\tau_{i j}^{\mathrm{a}}\right\rangle=(1-\phi)\left\langle\tau_{i j}^{\mathrm{s} 0}\right\rangle
$$

The magnitude of $\left\langle\tau_{i j}^{\mathrm{a}}\right\rangle$ differs from the in-situ stress by magnitude $\phi \delta_{i j} P_{\mathrm{p}}$. Expression (27) means that the effect of pore pressure on the average stress is equal for the normal stress in every direction independent of pore geometry. Therefore, the stress at the quasi-uniform state is independent of the elastic anisotropy of rock specimens, even if the anisotropy is caused by preferably oriented pores.

The contribution of $\phi P_{\mathrm{p}}$ is about $5 \%$ of the lithostatic pressure in magnitude, even for rocks of which the porosity is about $10 \%$, provided that $P_{\mathrm{p}}$ is the hydrostatic pressure at the depth. The contribution of pore pressure is comparable to the errors in magnitude in stress measurements. Thus, the effect may be negligible in general. However, the pore effect must be taken into consideration in the case of rocks beneath the seafloor because pore pressure is not small compared with the overburden pressure.

Tamaki et al. (1991) and Tamaki and Yamamoto (1992) measured the stresses on rocks beneath the seafloor by DRA. The strain difference functions obtained from the specimens showed profoundly curvilinear behavior, probably because of the small fracture strength of specimens for 
uni-axial compression. Although there may be some ambiguities in the identification of stress memory for the above reason, the folding could not be found near the overburden pressure calculated from the logging data, but near the applied stress, with the latter being smaller than the calculated overburden pressure by approximately the amount of $\phi P_{\mathrm{c}}$. This result suggests that Eq. (27) holds or that the measured magnitudes of stress are the absolute magnitudes of the stress to which the solid parts of rocks have been subjected at depths.

\section{Summary of the Mechanisms of In-situ Stress Memory}

In this paper, I have reviewed deformation rate analysis, which is a method developed for detecting in-situ stress memory in the inelastic deformation behavior of rock specimens under uni-axial loading of compression and demonstrated evidence for the existence of the rock property of in-situ stress memory. In order to elucidate this property, I have hypothesized that the stress field in rocks is quasiuniform at the in-situ stress at a depth. Here, the quasiuniform stress field means the field where stress concentration has partly relaxed. Therefore, the inelastic strain of rocks under axial loading consists of two modes of inelastic strain, Mode-I and Mode-II. The inelastic strain of Mode-I is caused by stress concentrations that increase with an increase in applied stress difference, a process that is well known. The inelastic strain of Mode-II is caused by the stress concentrations that increase with the deviation in the applied stress field from the quasi-uniform stress field.

The magnitude of stress concentrations is quantified by defining "stress deviation" in order to discuss the inelastic strain behavior of Mode-II, where the large "stress deviation" means the large magnitude of stress concentrations. The results theoretically derived from the above hypothesis are summarized as follows. (1) In rock specimens under the loading of uni-axial or tri-axial stress of compression, the "stress deviation" is minimized for all of the stress components, when the applied axial stress is equal in magnitude to the in-situ stress in the loading direction. (2) The "stress deviation" in a rock specimen under atmospheric pressure increases with an increase in the sampling depth because the average magnitude of in-situ stress increases with depth. (3) With hydrostatic pressure loading, we possibly find the applied pressure that minimizes the deviation of the normal component of stress for every direction. However, the pressure does not necessarily have the same magnitude as the in-situ stress of the direction because the deviation for a stress component reflects not only the in-situ stress of the component itself but also those of the other components in general.

Result (1) and the inferred behavior of different strain functions support the premise that the memory stress magnitudes measured by DRA are equal to those of in-situ stress in memory. Result (2) is in agreement with the existing observational results that crack porosity of rocks under the ambient condition increases in proportion to the sampling depth of the rocks.

\section{Suggestions for Further Study}

Rocks have been subjected for a long time to the insitu stress that is thought to be almost constant with time. The relaxation of stress concentrations may take place under such a constant stress as to form a less non-uniform stress field in rocks or to make the strain energy in rock decrease. The relaxation may be advanced by the partial transformation of shape or the deformation of constituent minerals that is slowly progressing by dislocation motions and/or by chemical reactions. As seen in this article, the proposed hypothesis appears to explain well the observed evidence, possibly indicating that the above process is actually progressing in nature.

The important problem to be solved is how long it takes for rocks to get in-situ stress memory. It has already been seen that vertical stress magnitudes correspond to the overburden pressures and that in-situ stress memory is observable a few years after recovering the rock samples from depths. These results provide the researcher with a rough idea of the time period when a rock has gotten the memory - that is, the time before a few years ago and after the formation of the topography. To further define this process, Yamamoto (1991) investigated the behavior of strain difference functions in relation to the loading duration by uni-axial loading of compression in laboratory. He found that the apparent compliance of a specimen becomes smaller around the applied stress as the loading duration increases. The apparent compliance may decrease due to the local relaxation of stress concentration. Such an approach may be useful for solving the problem of the time effect on the memory. Nevertheless, any reliable conclusion on the bounds has yet to be drawn. Further investigation is needed for this purpose.

The relationship between seismogenic stresses and stress distribution in the crust may be the key to revealing the conditions of earthquake generation. Although Yamamoto et al. (1997, 2004) discussed the conditions, their measurements were carried out at shallow depths of about $700 \mathrm{~m}$ at the most. Measurements at depths deeper than a few kilometers may be required to directly obtain information for this purpose. In this, I have theoretically clarified that insitu stresses at such great depths can be known in principle by applying DRA to the deformation of specimens under tri-axial loading of compression. However, the technology for the precise measurement of strain under confining pressures has not been established. This remains yet another problem for future technology to solve.

That the stress field in a rock is quasi-uniform at the insitu stress is currently considered to be a universal property of rocks. If the stress field is formed by the deformation of constituent minerals, the development of new methods for stress measurement at greater depths may be expected from the microscopic viewpoint. An investigation on the formation of the quasi-uniform stress field is required, not only to develop the new methods needed, but also to study the stress state of the Earth's crust.

Acknowledgments. I would like to express my deep felt thanks to two anonymous reviewers for their critical reading and thoughtful comments. Prof. Sano, ERI, Tokyo University and Dr. Ito, 
AIST, prepared the place for discussing the methods of in-situ stress measurement and their principles. I am extremely grateful to them. I would also like to express my gratitude to Honorary Prof. T. Hirasawa, Graduate School of Science, Tohoku University, Prof. K. Matsuki, Faculty of Technology, Tohoku University, and Dr. N. Kato, Tokyo University for their helpful suggestions and invaluable criticisms. I also heartily express my thanks to Dr. H. Yamamoto, Faculty of Technology, Iwate University for his help in laboratory experiments. This study was partly supported by the Grant-in-Aid for Scientific Research No. 04640396 (K. Yamamoto) and No. 04302052 (K. Matsuki).

\section{Appendix A. Stress Fields in Rocks as Composite Materials}

A.1 Definitions of macroscopically homogeneous and macroscopically uniform

Rocks are composite materials that consist of constituents such as mineral grains, fluid-filled pores, and voids. The constituents have various shapes and different physical properties. Let us suppose a small surface $\Delta \sigma$ including a point in a rock. We describe the traction acting on the surface by $\bar{\tau}_{j i} v_{j} \Delta \sigma \equiv \bar{\tau}_{j i} \Delta \sigma_{j}$, where $\bar{\tau}_{j i}$ is a stress component acting in the $x_{i}$ direction on a surface perpendicular to $x_{j}$, and $\Delta \sigma_{j}$ is the projection of $\Delta \sigma$ on the surface perpendicular to $x_{j}$. From the definition of stress (e.g., Sokolnikoff, 1956), $\bar{\tau}_{j i}$ is the $x_{i}$-component of the traction per unit area acting on the surface element $\Delta \sigma_{j}$. Here, we denote stress distributions in $\Delta \sigma_{j}$ by $\tau_{j i}(\boldsymbol{x})$ and divide $\Delta \sigma_{j}$ into a number of smaller surface elements, $d s_{j}$. Since $\bar{\tau}_{j i}$ are the average stresses on $\Delta \sigma_{j}$, the tractions $\bar{\tau}_{j i} \Delta \sigma_{j}$ are expressed by

$$
\begin{aligned}
\bar{\tau}_{j i} \Delta \sigma_{j} & =\int_{\Delta \sigma_{j}} \tau_{j i}(\boldsymbol{x}) d s_{j} \\
\Delta \sigma_{j} & =\int_{\Delta \sigma_{j}} d s_{j} .
\end{aligned}
$$

Therefore, we get

$$
\bar{\tau}_{j i}=\frac{1}{\Delta \sigma_{j}} \int_{d \Delta_{j}} \tau_{j i}(\boldsymbol{x}) d s_{j}
$$

Equation (A.1) or (A.2) denote that stresses $\bar{\tau}_{j i}$ can be defined on $\Delta \sigma_{j}$ without regard to the stress distribution on the surface $\Delta \sigma_{j}$.

If the average physical properties of a volume element are the same everywhere in a rock, the rock is said to be macroscopically homogeneous or "homogeneous". Therefore, a "homogeneous" material does not constrain the material to be homogenous from a microscopic viewpoint. If the average stresses $\bar{\tau}_{j i}$ in an element of a volume are constant everywhere in a rock, we say that the stress field is macroscopically uniform or "uniform". The "uniform" stresses $\tau_{j i}$ denote the averages of $\tau_{j i}(\boldsymbol{x})$ in a volume element that are constant anywhere in a rock. The "uniform" stress field thus does not constrain the stress field to be uniform from a microscopic viewpoint.

\section{A.2 Expressions for average stresses in a macroscopi- cally homogeneous body}

Suppose a rock of a unit volume bounded by a surface $S$. By applying the divergent theorem (the Gauss theorem) to the volume, the following equation is obtained;

$$
\begin{aligned}
\int_{S} x_{j} \check{T}_{i}(\boldsymbol{x}) d s & =\int_{S} x_{j} \tau_{k i}(\boldsymbol{x}) v_{k} d s=\int_{V}\left\{x_{j} \tau_{k i}(\boldsymbol{x})\right\}_{, k} d v \\
& =\int_{V}\left\{\tau_{j i}(\boldsymbol{x})+x_{j} \tau_{k i}(\boldsymbol{x})_{, k}\right\} d v
\end{aligned}
$$

where $\check{T}_{i}(\boldsymbol{x}) d s$ is the $i$-th component of the traction acting on a surface element $d s$ of $S$, of which the normal is parallel to the direction $\boldsymbol{v}$ and $v_{k}$ is the $k$-th component of $\boldsymbol{v}$. $\tau_{k i}(\boldsymbol{x})$ and $\tau_{k i}(\boldsymbol{x})_{, k}$ are stress components acting in the $x_{i}$ direction on a surface perpendicular to $x_{k}$ and its derivative with respect to $x_{k}$, respectively. $d v$ is a volume element. From (A.3), the following equation is derived:

$$
\begin{aligned}
\frac{1}{2} \int_{S}\left\{x_{j} \check{T}_{i}(\boldsymbol{x})+x_{i} \check{T}_{j}(\boldsymbol{x})\right\} d s= & \frac{1}{2} \int_{V}\left\{\tau_{j i}(\boldsymbol{x})+\tau_{i j}(\boldsymbol{x})\right. \\
& \left.+x_{j} \tau_{k i}(\boldsymbol{x})_{, k}+x_{i} \tau_{k j}(\boldsymbol{x})_{, k}\right\} d v
\end{aligned}
$$

The average stresses in the body are expressed by

$$
\left\langle\tau_{i j}\right\rangle=\left\langle\tau_{j i}\right\rangle=\frac{1}{2} \int_{V}\left\{\tau_{j i}(\boldsymbol{x})+\tau_{i j}(\boldsymbol{x})\right\} d v .
$$

When any part of the body is equilibrated, the following equation is obtained by employing the equilibrium equation for the right-hand side of (A.4):

$$
\begin{aligned}
\left\langle\tau_{i j}\right\rangle= & \frac{1}{2} \int_{S}\left(x_{j} \check{T}_{i}(\boldsymbol{x})+x_{i} \check{T}_{j}(\boldsymbol{x})\right) d s \\
& +\frac{1}{2} \int_{V}\left(x_{j} f_{i}(\boldsymbol{x})+x_{i} f_{j}(\boldsymbol{x})\right) d v
\end{aligned}
$$

Here, $f_{i}(\boldsymbol{x})$ are the body forces acting in the $x_{i}$ direction at a point $\boldsymbol{x}$. A similar expression for the composite materials that are free from body forces is described in a text by Landau and Lifshitz (1972) and in a paper by Hill (1963). In this derivation of (A.5), nothing is assumed, except for the assumption that the equilibrium equation holds and the stress field is differentiable. We denote $x_{j} f_{i}(\boldsymbol{x})+x_{i} f_{j}(\boldsymbol{x})$ as internal stresses.

\section{A.3 Expressions for the stress field in a body under macroscopically uniform stresses}

If the surface tractions and the body forces are independent from each other, the stresses in the body may be expressed by the superposition of the stresses produced by the applied stresses on $S$ and the stresses caused by the internal stresses, that is,

$$
\tau_{i j}(\boldsymbol{x})=\tau_{i j}^{*}(\boldsymbol{x})-\tau_{i j}^{\mathrm{c}}(\boldsymbol{x}),
$$

where $\tau_{i j}^{*}(\boldsymbol{x})$ and $\tau_{i j}^{\mathrm{c}}(\boldsymbol{x})$ are the stresses due to the applied stress and the stresses due to body forces, respectively. The resultants of surface tractions $\breve{T}_{j}(\boldsymbol{x})$ on $S$ should be equilibrated to body force $-F_{j}$ from the outer field, that is,

$$
\begin{aligned}
\int_{S} \check{T}_{j}(\boldsymbol{x}) d s & =\int_{S}\left(\tau_{i j}^{*}(\boldsymbol{x})-\tau_{i j}^{\mathrm{c}}(\boldsymbol{x})\right) v_{i} d s \\
& =\int_{V} \tau_{i j}^{*}(\boldsymbol{x})_{, i} d v-\int_{V} \tau_{i j}^{\mathrm{c}}(\boldsymbol{x})_{, i} d v=-F_{j}
\end{aligned}
$$


From the equilibrium equation and the definitions of $\tau_{i j}^{*}(\boldsymbol{x})$ and $\tau_{i j}^{\mathrm{c}}(\boldsymbol{x})$, we may write

$$
\begin{aligned}
\int_{V} \tau_{i j}^{*}(\boldsymbol{x})_{, i} d v & =0 \\
\int_{V} \tau_{i j}^{\mathrm{c}}(\boldsymbol{x})_{, i} d v & =\int_{V} f_{j}(\boldsymbol{x}) d v=F_{j} .
\end{aligned}
$$

In the case of $F_{j}=0, f_{j}(\boldsymbol{x})$ are interpreted as internal forces. From (A.8), we can take (A.6) as the general equation that represents the stress field in rocks including internal forces.

\section{A.4 Distribution of internal stresses}

Consider the case that the spatial and the magnitude distributions of $f_{i}(\boldsymbol{x})$ are independent of each other and respectively random in a rock body. We put

$$
\begin{aligned}
f_{i}(\boldsymbol{x}) & =f_{i}^{0}+\delta f_{i}(\boldsymbol{x}) \\
x_{j} & =x_{j}^{0}+\delta x_{j},
\end{aligned}
$$

where

$$
\begin{aligned}
f_{i}^{0} & =\left\langle f_{i}(\boldsymbol{x})\right\rangle=\int_{V} f_{i}(\boldsymbol{x}) d v \\
x_{j}^{0} & =\left\langle x_{j}\right\rangle=\int_{V} x_{j} d v \\
\left\langle\delta f_{j}(\boldsymbol{x})\right\rangle & =\int_{V} \delta f_{j}(\boldsymbol{x}) d v=0 \\
\left\langle\delta x_{j}\right\rangle & =\int_{V} \delta x_{j} d v=0 .
\end{aligned}
$$

By making use of (A.9) and (A.10), the following equation is obtained,

$$
\begin{aligned}
\int_{V} x_{i} f_{j}(\boldsymbol{x}) d v= & \int_{V}\left(x_{i}^{0}+\delta x_{i}\right)\left(f_{j}^{0}+\delta f_{j}(\boldsymbol{x})\right) d v \\
= & \int_{V}\left(x_{i}^{0} f_{j}^{0}+x_{i}^{0} \delta f_{j}(\boldsymbol{x})+f_{j}^{0} \delta x_{i}\right. \\
& \left.+\delta x_{i} \delta f_{j}(\boldsymbol{x})\right) d v
\end{aligned}
$$

Since the spatial distribution and the magnitude distribution of $f_{j}(\boldsymbol{x})$ are independent of each other,

$$
\left\langle\delta x_{i} \delta f_{j}(\boldsymbol{x})\right\rangle=\left\langle\delta x_{i}\right\rangle\left\langle\delta f_{j}(\boldsymbol{x})\right\rangle=0 .
$$

When $f_{j}(\boldsymbol{x})$ are internal forces, $f_{j}^{0}=0$. Therefore, from (A.11) and (A.12)

$$
\int_{V} x_{i} f_{j}(\boldsymbol{x}) d v=0 .
$$

As shown above, when the spatial and magnitude distribution of internal forces are random and independent of each other, the second term on the right-hand side of (4a) in the text is zero.

Equations (A.1) or (A.2) mean that the Eqs. (A.5) and (A.6) are valid for the macroscopically uniform stress field, including the non-uniform stresses produced by nonhomogeneity of materials from the microscopic viewpoint. In this article, the macroscopically uniform stress field has been discussed. Therefore, discussions based on (A.5) or (A.6) are, in general, valid for the macroscopically uniform stress field in macroscopically homogeneous rocks.

\section{Appendix B. Behavior of Strain Difference Func- tions}

\section{B.1 Expressions of strain difference functions}

When cyclic axial loading of compression is performed for a rock specimen, the relationship between applied axial stress $\sigma_{1}$ and axial strain $\varepsilon_{k}\left(\sigma_{1}\right)$ during $k$-th loading may be expressed from expression (10) in the text by

$$
\varepsilon_{k}\left(\sigma_{1}\right)=\varepsilon_{k}^{\mathrm{el}}\left(\sigma_{1}\right)+(1-\eta) \varepsilon_{k}^{\mathrm{I}}\left(\sigma_{1}\right)+\eta \varepsilon_{k}^{\mathrm{II}}\left(\sigma_{1}\right),
$$

where $\varepsilon_{k}^{\mathrm{el}}\left(\sigma_{1}\right)$ is the elastic strain. The strain difference function $\Delta \varepsilon_{k, k+1}\left(\sigma_{1}\right)$ used in the DRA is written by

$$
\begin{aligned}
\Delta \varepsilon_{k, k+1}\left(\sigma_{1}\right)= & \Delta \varepsilon_{k, k+1}^{\mathrm{el}}\left(\sigma_{1}\right)+(1-\eta) \Delta \varepsilon_{k, k+1}^{\mathrm{I}}\left(\sigma_{1}\right) \\
& +\eta \Delta \varepsilon_{k, k+1}^{\mathrm{II}}\left(\sigma_{1}\right) \quad \text { for } 0 \leq \sigma_{1} \leq \sigma_{1}^{\mathrm{P}} \\
\Delta \varepsilon_{k, k+1}^{\mathrm{el}}\left(\sigma_{1}\right)= & \varepsilon_{k+1}^{\mathrm{el}}\left(\sigma_{1}\right)-\varepsilon_{k}^{\mathrm{el}}\left(\sigma_{1}\right) \\
\Delta \varepsilon_{k, k+1}^{\mathrm{I}}\left(\sigma_{1}\right)= & \varepsilon_{k+1}^{\mathrm{I}}\left(\sigma_{1}\right)-\varepsilon_{k}^{\mathrm{I}}\left(\sigma_{1}\right) \\
\Delta \varepsilon_{k, k+1}^{\mathrm{II}}\left(\sigma_{1}\right)= & \varepsilon_{k+1}^{\mathrm{II}}\left(\sigma_{1}\right)-\varepsilon_{k}^{\mathrm{II}}\left(\sigma_{1}\right) .
\end{aligned}
$$

Here, $\sigma_{1}^{\mathrm{P}}$ is the peak of the applied stress.

The crack model by Kuwahara et al. (1990) described in Section 2.1 may be generalized as one of the models to explain the mechanisms that release the shear stress concentration in rocks. In the present discussion, the model is adopted to elucidate the inelastic deformation. The following are assumed when the crack model (Kuwahara et al., 1990) is taken into account: (1) inelastic strain increases with an increase in the absolute value of the stress deviation; (2) inelastic strain is increased by shear cracks accompanied with tensile cracks produced by micro-fracturing; (3) new micro-fracturing begins to occur at the point where the value of applied axial stress reaches the peak value of the stress previously applied to the specimens; (4) the activity of micro-fracturing gradually increases with an increase in the applied stress for an applied stress larger than the previously applied peak stress; (5) the pre-existing micro-cracks stably slide in response to applied stress. It is additionally assumed here that (6) the activity of micro-fracturing decreases with time under a constant applied stress or with the number of cycles under cyclic loading with a constant peak stress. Assumption (6) is likely to hold for an applied axial stress smaller than the stress that causes dilatancy.

When the change in elastic constants of specimens with an increase in loading cycle is negligibly small, $\Delta \varepsilon_{k, k+1}^{\mathrm{el}}\left(\sigma_{1}\right)$ in (B.2) may be neglected. Therefore, $\Delta \varepsilon_{k, k+1}\left(\sigma_{1}\right)$ is considered to mainly represent the difference in the behavior of the inelastic strain for the loading stage between the cycles, that is,

$$
\Delta \varepsilon_{k, k+1}\left(\sigma_{1}\right)=(1-\gamma) \Delta \varepsilon_{k, k+1}^{\mathrm{I}}\left(\sigma_{1}\right)+\gamma \Delta \varepsilon_{k, k+1}^{\mathrm{II}}\left(\sigma_{1}\right) .
$$

In order to discuss the behavior of strain difference function in response to applied stress, it is necessary to specify the relationship of the inelastic strain and applied stress.

In the case of Mode-I

Here, the inelastic strain of Mode-I for the loading stage of the $k$ th loading cycle is assumed to be expressed by a 
series of power functions of $\sigma_{1}$,

$$
\varepsilon_{k}^{\mathrm{I}}\left(\sigma_{1}\right)=\alpha_{k}+\alpha_{k}^{0} \sigma_{1}+\sum_{l=1} \alpha_{k}^{l} \sigma_{1}^{l} \quad \text { for } 0 \leq \sigma_{1} \leq \sigma_{1}^{\mathrm{P}},
$$

where $l$ is an integer of 1 to an arbitrary number, and $\alpha_{k}$ and $\alpha_{k}^{l}$ are constants. The first two terms of the right-hand side are the residual strain and the inelastic strains, respectively, due to the deformation of existing micro-cracks in response to applied stress. The other term represents the amount of inelastic strain newly produced when the applied stress is increased to $\sigma_{1}$ from 0 in the $k$ th loading.

In the first loading, micro-fracturing occurs at an increasing activity with $\sigma_{1} \cdot \varepsilon_{1}^{\mathrm{I}}\left(\sigma_{1}\right)$ is thus expected to be continuous and a monotonously increasing function of $\sigma_{1}$. Since almost all of the potential micro-fractures are produced in the first loading, the stress-strain relations in the subsequent loading cycles are more linear than those of the first loading according to assumption (5). The gradients of the linear component in the subsequent loadings may be much larger than that of the first loading and be almost constant because an increase in cracks produced in the subsequent loading cycles may be negligibly smaller than that in the first loading. Therefore, when assumption (6) is taken into account, the following equations may hold,

$$
\begin{array}{ll}
\alpha_{1}^{0} \ll \alpha_{k}^{0} \leq \alpha_{k+1}^{0} & \text { for } k \neq 1 \\
\alpha_{k+1}^{l} \leq \alpha_{k}^{l} \ll \alpha_{1}^{l} & \text { for } k \neq 1 .
\end{array}
$$

The strain difference function may be approximately written as

$\Delta \varepsilon_{1,2}^{\mathrm{I}}\left(\sigma_{1}\right) \approx\left(\alpha_{2}-\alpha_{1}\right)+\alpha_{2}^{0} \sigma_{1}-\sum_{l=1} \alpha_{1}^{l} \sigma_{1}^{l}$ for $0 \leq \sigma_{1} \leq \sigma_{1}^{\mathrm{P}}$,

for $k=1$ and

$$
\begin{aligned}
\Delta \varepsilon_{k, k+1}^{\mathrm{I}}\left(\sigma_{1}\right) \approx & \left(\alpha_{k+1}-\alpha_{k}\right)+\left(\alpha_{k+1}^{0}-\alpha_{k}^{0}\right) \sigma_{1} \\
& +\sum_{l=1}\left(\alpha_{k+1}^{l}-\alpha_{k}^{l}\right) \sigma_{1}^{l} \quad \text { for } 0 \leq \sigma_{1} \leq \sigma_{1}^{\mathrm{P}},
\end{aligned}
$$

for $k \neq 1$. From (B.5), $\left(\alpha_{k+1}^{0}-\alpha_{k}^{0}\right)$ and $\left(\alpha_{k+1}^{l}-\alpha_{k}^{l}\right)$ may be a positive small value and a negative small value, respectively.

In the case of Mode-II

When rocks are brought from depths to ground surface by coring, $I_{i}$ for the Mode-II may increase with an increase in $\left|\sigma_{1}-\tau_{1}^{0}\right|$ to generate micro-fractures to some extent. The stress measurement is completed for negligibly short duration compared with the time interval from the coring to the measurement. Cored rocks are laid out under atmospheric pressure during the time interval. It may be considered that almost all of the potential micro-fractures due to $I_{i}$ for the Mode-II have fractured during the interval. For this reason, a negligibly small number of micro-fractures occur for $\sigma_{1}$ from 0 to $\tau_{1}^{0}$ for all loading cycles. On the other hand, for $\tau_{1}^{0}<\sigma_{1} \leq \sigma_{1}^{\mathrm{P}}$ the micro-fracturing may occur similarly to that in the case of the Mode-I. Here, the inelastic strain of
Mode-II for $k$ th loading is assumed to be written by

$$
\begin{array}{r}
\varepsilon_{k}^{\mathrm{II}}\left(\sigma_{1}\right)=\gamma^{\prime}+\gamma\left(\sigma_{1}-\tau_{1}^{0}\right) \quad \text { for } 0 \leq \sigma_{1}<\tau_{1}^{0} \\
\varepsilon_{k}^{\mathrm{II}}\left(\sigma_{1}\right)=\beta_{k}+\beta_{k}^{0}\left(\sigma_{1}-\tau_{1}^{0}\right)+\sum_{l=1} \beta_{k}^{l}\left(\sigma_{1}-\tau_{1}^{0}\right)^{l} \\
\text { for } \tau_{1}^{0} \leq \sigma_{1} \leq \sigma_{1}^{\mathrm{P}}
\end{array}
$$

where $\gamma, \gamma^{\prime}$, and $\beta_{k}^{l}$ are constants. The first two terms of the right-hand side of both equations in (B.8) are the contributions from the existing micro-cracks. For the same reasons as those for Mode-I, the following relationships may hold,

$$
\begin{array}{ll}
\beta_{1}^{0} \ll \beta_{k}^{0} \leq \beta_{k+1}^{0} & \text { for } k \neq 1 \\
\beta_{k+1}^{l} \leq \beta_{k}^{l} \ll \beta_{1}^{l} & \text { for } k \neq 1
\end{array}
$$

The strain difference during loading may be written as follows:

$$
\begin{aligned}
& \Delta \varepsilon_{1,2}^{\mathrm{II}}\left(\sigma_{1}\right) \approx 0 \quad \text { for } 0 \leq \sigma_{1}<\tau_{1}^{0} \\
& \Delta \varepsilon_{1,2}^{\mathrm{II}}\left(\sigma_{1}\right) \approx\left(\beta_{2}-\beta_{1}\right)+\beta_{2}^{0}\left(\sigma_{1}-\tau_{1}^{0}\right)-\sum_{l=1} \beta_{1}^{l}\left(\sigma_{1}-\tau_{1}^{0}\right)^{l} \\
& \quad \text { for } \tau_{1}^{0} \leq \sigma_{1} \leq \sigma_{1}^{\mathrm{P}},
\end{aligned}
$$

for $k=1$ and

$$
\begin{aligned}
& \Delta \varepsilon_{k, k+1}^{\mathrm{II}}\left(\sigma_{1}\right) \approx 0 \quad \text { for } 0 \leq \sigma_{1}<\tau_{1}^{0} \\
& \begin{aligned}
\Delta \varepsilon_{k, k+1}^{\mathrm{II}}\left(\sigma_{1}\right) \approx & \left(\beta_{k+1}-\beta_{k}\right)+\left(\beta_{k+1}^{0}-\beta_{k}^{0}\right)\left(\sigma_{1}-\tau_{1}^{0}\right) \\
& +\sum_{l=1}\left(\beta_{k+1}^{l}-\beta_{k}^{l}\right)\left(\sigma_{1}-\tau_{1}^{0}\right)^{l} \\
& \quad \text { for } \tau_{1}^{0} \leq \sigma_{1} \leq \sigma_{1}^{\mathrm{P}}
\end{aligned}
\end{aligned}
$$

for $k \neq 1$. From (B.9), $\left(\beta_{k+1}^{0}-\beta_{k}^{0}\right)$ and $\left(\beta_{k+1}^{l}-\beta_{k}^{l}\right)$ may be a positive small value and a negative small value, respectively.

\section{B.2 Behavior of strain difference functions}

The gradient change of a strain difference function given by the inelastic strain of Mode-II is important for DRA. For $k=1$, even if $\Delta \varepsilon_{k, k+1}^{\mathrm{II}}\left(\sigma_{1}\right)$ discontinuously changes at $\sigma_{1}=\tau_{1}^{0}$, the change may, in general, be buried in the huge curvilinear behavior of the strain difference function of Model-I. For $k \neq 1, \partial \Delta \varepsilon_{k, k+1}^{\mathrm{II}}\left(\sigma_{1}\right) / \partial \sigma_{1}<0$ for $\tau_{1}^{0} \leq$ $\sigma_{1} \leq \sigma_{1}^{\mathrm{P}}$. Therefore, the following inequality is obtained from expression (B.11),

$$
\left(\beta_{k+1}^{0}-\beta_{k}^{0}\right)<\left(\beta_{k}^{1}-\beta_{k+1}^{1}\right)+\sum_{l=2} l\left(\beta_{k}^{l}-\beta_{k+1}^{l}\right)\left(\sigma_{1}-\tau_{1}^{0}\right)^{l-1} .
$$

The coefficient $\left(\beta_{k+1}^{0}-\beta_{k}^{0}\right)$ represents the increase in the gradient caused by the deformation of the existing cracks produced in the $k$ th loading. If the existing cracks are completely locked, the produced inelastic strain during loading is not recovered at all during unloading, and $\left(\beta_{k+1}^{0}-\beta_{k}^{0}\right)=0$ is expected. The offset $\left(\beta_{k+2}-\beta_{k+1}\right)$ of strain after the $(k+1)$-th cycle of loading and unloading is equal to the sum of the inelastic strain produced and that recovered in the cycle. When the pre-existing cracks are completely locked, 
$\left(\beta_{k+2}-\beta_{k+1}\right)=\Delta \varepsilon_{k, k+1}^{\mathrm{II}}\left(\sigma_{1}^{\mathrm{P}}-\tau_{1}^{0}\right)$. Although the offset has not been quantitatively analyzed, the observation of the reduced stress-strain relations suggests that the recovery appears to be small. For the reason above, $\left(\beta_{k+1}^{0}-\beta_{k}^{0}\right)$ may be neglected here. In this case, the step-like gradient change should be observed so long as the step by $\left(\beta_{k}^{1}-\beta_{k+1}^{1}\right)$ is not buried in the curvilinear behavior of the inelastic strain of Mode-I. In the case that $\left(\beta_{k+1}^{1}-\beta_{k}^{1}\right)$ is small, a gradual change in the gradient $\left(\beta_{k}^{l}-\beta_{k+1}^{l}\right)$ for $l \geq 2$ may be observed.

\section{References}

Brace, W. F., B. W. Paulding, and C. H. Scholz, Dilatancy in the fracture of crystalline rocks, J. Geophys. Res., 71, 3939-3953, 1966.

Carlson, S. and H. Wang, Microcrack porosity and in situ stress in Illinois Borehole UPH-3, J. Geophys. Res., 91, 10,421-10,428, 1986.

Enever, J. and L. Mckay, Note of the relationship between anelastic strain recovery and virgin rock stresses - a possible method of stress measurement, ISRM Symp. on Investigation of Stresses in Rock-Advance in Stress Measurement, Sydney, 37-40, 1976.

Goodman, R. E., Subaudible noise during compression of rocks, Geol. Soc. Am. Bull., 74, 487-490, 1963.

Hill, R., Elastic properties of rein forced solid: Some theoretical principles, J. Mech. Phys. Solids, 11, 357-372, 1963.

Kanagawa, T., M. Hayashi, and N. Nakasa, Estimation of spatial geostress components in rock samples using the Kaiser effect of acoustic emission, Proc. Jpn. Soc. Civil Eng., 285, 63-75, 1977 (in Japanese).

Koide, H., Y. Nishimatsu, S. Koizumi, K. Hoshino, T. Kanagawa, Y. Nakayama, A. Inoue, K. Yamamoto, and S. Kikuchi, Comparison among several methods for stress measurement in the Kanto-Tokai district, Japan, Proc. 18th Japan Symp. Rock Mech., 261-265, 1986 (in Japanese with English abstract).

Kurita, K. and N. Fujii, Stress memory of crystalline rocks in acoustic emission, Geophys. Res. Lett., 6, 9-12, 1979.

Kuwahara, Y., K. Yamamoto, and T. Hirasawa, An experimental and theoretical study of inelastic deformation of brittle rocks under cyclic uniaxial loading, Tohoku Geophys. J. (Sci. Rep. Tohoku Univ., Ser. 5), 33, $1-21,1990$.

Landau, L. and E. Lifshitz, Theory of Elasticity, New edition with supplements, 1989, translated in Japanese by Sato, T. and Y. Ishibashi, Tokyo Tosho Co., Bunkyo-ku, Tokyo, pp 275, 1972.

Meglis, I. L., T. Engelder, and E. K. Graham, The effect of stress-relief on ambient microcrack porosity in core samples from the Kent Cliffs (New York) and Moodus (Connecticut) scientific research boreholes, Tectonophysics, 186, 163-173, 1991.

Montgomery, C. T. and N.-K. Ren, Differential strain curve analysis: Does it work ?, Proc. Workshop on hydraulic fracturing stress measurement, Monterey, CA, 239-245, 1981.

Sato, N., Y. Yabe, K. Yamamoto, and H. Ito, In situ stresses near the Nojima Fault estimated by deformation rate analysis, Zisin 2, 56, 157$169,2003$.
Seto, M., D. K. Nag, and V. S. Vutukuri, In-situ rock stress measurement from rock cores using the acoustic estimation method and deformation rate analysis, Geotech. Geol. Eng., 17, 241-266, 1999.

Simmons, G., R. W. Siegfried II, and M. Faves, Differential strain analysis: A new method for examining cracks in rocks, J. Geophys. Res., 79, 4383-4385, 1974.

Sokolnikoff, I. S., Mathematical Theory of Elasticity, McGraw-Hill, New York, pp. 476, 1956.

Tamaki, K. and K. Yamamoto, Estimating in-situ stress field from basaltic rock core samples of Hole 794C, Yamato Basin, Japan Sea, Proceedings of the Ocean Drilling Program, Scientific Results, 127/128, 1047-1059, 1992.

Tamaki, K., K. Yamamoto, T. Furuta, and H. Yamamoto, An experiment of in-situ stress estimation on basaltic core samples from Hole 758A, Ninetyeast Ridge, Indian Ocean, Proceedings of the Ocean Drilling Program, Scientific Results, 121, 697-917, 1991.

Yamamoto, H., An experimental study on stress memory of rocks and its application to in situ stress estimation, Master thesis, Tohoku Univ., pp. 96, 1991.

Yamamoto, K., The rock property of in-situ stress memory: Discussions on its mechanism, in Proc. Int. W/S Rock Stress Measurement at Great Depth, edited by Matsuki, K. and K. Sugawara, 46-51, ISRM, Tokyo, 1995.

Yamamoto, K. and Y. Yabe, Stresses at sites close to the Nojima earthquake fault measured on boring core samples, Island Arc, 10, 266-281, 2001.

Yamamoto, K., Y. Kuwahara, and T. Hirasawa, Discrimination of previously applies stress by the deformation rate analysis: Application of the method to estimation of in-situ stress, Progr. Abstr. Seismol. Soc. Jpn., 1983, 2:104, 1983 (Abstract in Japanese).

Yamamoto, K., Y. Kuwahara, N. Kato, and T. Hirasawa, Deformation rate analysis: A new method for in situ stress estimation from inelastic deformation of rock samples under uni-axial compressions, Tohoku Geophys. J. (Sci. Rep. Tohoku Univ., Ser. 5), 33, 127-147, 1990.

Yamamoto, K., H. Yamamoto, N. Kato, and T. Hirasawa, Deformation rate analysis for in situ stress estimation, in AE/MS Activity in Geol. Struc. Mat. (Proc. of the Fifth Conference), edited by H. R. Hardy, Jr, 243-255, Trans Tech Pub, 1995.

Yamamoto, K., Y. Yabe, and H. Yamamoto, Relation of In-situ stress field to seismic activity as inferred from the stresses measured on core samples, in Rock Stress (Proc. Int. Symp. Rock Stress, Kumamoto/Japan/710 October, 1977), edited by Sugawara, K. and Y. Obara, 375-380, Balkema, Rotterdam, 1997.

Yamamoto, K., N. Sato, and Y. Yabe, Driving force of the intra-plate crust as inferred from stresses measured in the eastern part of Kitakami mountains, Northeastern Honshu, Japan, Zisin 2, 56, 511-527, 2004 (in Japanese with English abstract).

Yoshikawa, S. and K. Mogi, A new method for estimation of the crustal stress from cored rock samples: Laboratory study in the case of uniaxial compression, Tectonophysics, 74, 323-339, 1981.

K. Yamamoto (e-mail: fwiz8233@nifty.com) 